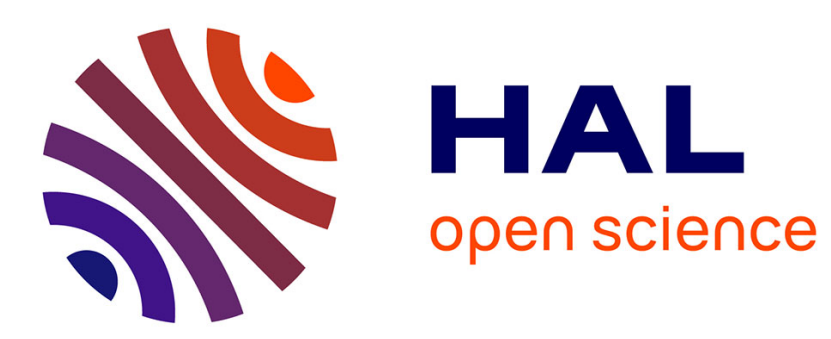

\title{
Allocation Rules for Multi-choice Games with a Permission Tree Structure
}

David Lowing

\section{To cite this version:}

David Lowing. Allocation Rules for Multi-choice Games with a Permission Tree Structure. 2021. halshs-03121514

\section{HAL Id: halshs-03121514 https://shs.hal.science/halshs-03121514}

Preprint submitted on 26 Jan 2021

HAL is a multi-disciplinary open access archive for the deposit and dissemination of scientific research documents, whether they are published or not. The documents may come from teaching and research institutions in France or abroad, or from public or private research centers.
L'archive ouverte pluridisciplinaire HAL, est destinée au dépôt et à la diffusion de documents scientifiques de niveau recherche, publiés ou non, émanant des établissements d'enseignement et de recherche français ou étrangers, des laboratoires publics ou privés. 
UMR 5824

93. chemin des Mouilles 69130 Ecully - France

Maison de IUniversité, Bâtiment $\mathrm{B}$ 10, rue Trefilerie 42023 Saint-Etienne cedex $02 \cdot$ France http://www.gate.cnrs.fr gate@gate.cnrs.fr

WP 2106 - January 2021

\title{
Allocation Rules for Multi-choice Games with a Permission Tree Structure
}

David Lowing

\begin{abstract}
:
We consider multi-choice cooperative games with a permission tree structure. Multi-choice games are a generalization of a cooperative transferable utility games in which each player has several activity levels. In addition, a permission tree structure models a situation in which a player needs permission from another player to cooperate. In this framework, the influence of a permission structure on the possibility of cooperation may have several interpretations depending on the context. In this paper, we investigate several of these interpretations and introduce for each of them a new allocation rule that we axiomatically characterize..
\end{abstract}

Keywords:

Multi-choice games, Multi-choice Permission value, Permission (tree) structures

JEL codes:

C71 


\title{
Allocation Rules for Multi-choice Games with a Permission Tree Structure.
}

\author{
David Lowing*†
}

2021

\begin{abstract}
We consider multi-choice cooperative games with a permission tree structure. Multichoice games are a generalization of a cooperative transferable utility games in which each player has several activity levels. In addition, a permission tree structure models a situation in which a player needs permission from another player to cooperate. In this framework, the influence of a permission structure on the possibility of cooperation may have several interpretations depending on the context. In this paper, we investigate several of these interpretations and introduce for each of them a new allocation rule that we axiomatically characterize.

Keywords: Multi-choice games $\cdot$ Multi-choice Permission value $\cdot$ Permission (tree) structures

JEL codes: C71
\end{abstract}

\section{Introduction}

A situation in which a finite set of players can generate a certain payoff by cooperating can be described through a cooperative game with transferable utility (henceforth TU-game). To accurately model some real-life situations, several extensions of TU-games have been proposed. In this paper, we consider two generalizations of TU-games: multi-choice games and TU-games equipped with a structure defined on the player set.

In TU-games, players choose whether or not to join a project involving cooperation with several agents. Multi-choice cooperative games are introduced by Hsiao and Raghavan 1992

*Univ Lyon, UJM Saint-Etienne, GATE Lyon Saint-Etienne UMR 5824, F-42023 Saint-Etienne, France.

${ }^{\dagger}$ Email: david.lowing@grdf.fr 
to model cooperative situations where each player has a finite set of activity levels from nonparticipation (a participation level 0) to a maximal participation level. Several extensions of solutions from TU-games to multi-choice games can be found, among others, in van den Nouweland et al. |PhD thesis, Tilburg University, 1993], Derks and Peters [1993], Klijn et al. [1999], Calvo and Santos [2000], Peters and Zank [2005], Grabisch and Lange [2007] and Branzei et al. [2009], and Branzei et al. [2014].

In the classical model of TU-games, it is also generally assumed that cooperation among group of players is not constrained. However, in practice, several structures have an impact on the formation or evaluation of coalitions. TU-games endowed with a structure on the player set have been introduced to model situations where the formation or evaluation of coalitions depends on communicational, coalitional or hierarchical constraints. For instance, communicational constraints, modeled by an undirected graph, are discussed in Myerson [1977]. Players are located on the vertices of an undirected graph where the set of edges represents the communication channels between pair of players. A coalition of players can cooperate only if they can communicate through a path that lies in this coalition. Coalitional constraints where players meet together in coalitions and form a priori unions are modeled by a partition of the player set and discussed in Aumann and Dreze [1974] and Owen [1977]. Different interpretations of this model are possible. In Aumann and Dreze [1974] players belonging to different a priori unions can not cooperate whereas in Owen [1977| the formation of a coalition must be consistent with the structure of a priori unions. Hierarchical constraints, as modeled by Gilles et al. [1992] and van den Brink and Gilles [1996], describe situations in which players need the permission from other players before they are allowed to cooperate within a coalition. These hierarchical constraints are modeled by a permission structure represented by a directed graph on the player set.

In this paper we take into account the above two generalizations by considering multichoice games and structures on the player set simultaneously. Indeed, it is natural to assume that players can both cooperate at different activity levels and be constrained by external factors in their attempts to form coalitions. A few studies have been conducted in this direction. For instance, Béal et al. 2012] combine communicational constraints and multi-choice games and axiomatically characterize an allocation rule which generalizes the Average tree solution introduced by Herings et al. [2008] from TU-games enriched by a communication graph to multi-choice games enriched by a communication graph. Albizuri [2009] combines multi-choice games with a priori unions and axiomatically characterize an allocation rule which generalizes the Owen value (Owen [1977]) from TU-games with a priori unions to multi-choice games with a priori unions. We propose to enrich the model of multi-choice games with a permission structure and to investigate how hierarchical constraints on the player set can be combined with different levels of participations. There is no straightforward way to combine these two aspects. We propose to deal with this problem in two steps which correspond to two approaches describing two different but closely related models. In a first approach, we consider multi-choice games enriched by a permission structure on the set of player-activity levels pairs $M^{+}$. In a second approach, we consider multi-choice games with a hierarchy on the set of players. 
(a) The first approach consists in defining a permission structure on the player-activity level pairs (pal-permission henceforth) and not on the player set as in Gilles et al. [1992]. The underlying idea is that a player $i$ making a certain activity level $j$ needs the permission of another player $k$ making a certain activity level $l$ before to cooperate. This approach generalizes in a straightforward way the model introduced by Gilles et al. [1992]. We proceed in several steps. First, we define a set of feasible coalitions with respect to a pal-permission structure and show that this set forms a sub-lattice of the set of coalitions. Second, we define the sovereign and authorizing parts of a coalition $s$ as its largest feasible sub-coalition and its smallest feasible super-coalition, respectively. Third, we introduce a restricted multichoice game given by the worths of the sovereign part of the coalitions. Finally, we define a Multi-choice permission value that generalizes both the Derks-Peters value (Derks and Peters [1993]) for multi-choice games and the Permission value for TU-games with a permission structure (Gilles et al. [1992 and van den Brink and Gilles 1996]), where both the Derks-Peters value and the Permission value are generalizations of the Shapley value (Shapley [1953]) from TU-games to multi-choice games and from TU-games to TU-games with a permission structure, respectively. To be precise, we apply the Derks-Peters value to the above restricted multi-choice game.

(b) The second approach keeps a permission structure on the player set, as in Gilles et al. [1992], and to combine it with a multi-choice game. We restrict ourselves to a permission structure on the player set that can be represented by a rooted tree. In this second approach, it is always considered that the players are part of a hierarchical structure but we also exploit the fact that the set of activity levels of each player is totally ordered. This leads us to consider that there are two types of permission structures: the first one operates between players and the second one operates between the activity level of each player. Indeed, one considers that the activity levels are part of a hierarchical structure in the sense that choosing the activity level $j$ requires having made or being able to make the activity level $j-1$. In other words, players gradually deploy their activity levels, from the lowest to the highest level.

We propose several axioms which allows us to characterize three new allocation rules. Each one coincides with a Multi-choice permission value on multi-choice games enriched with a pal-permission structure as described in the first approach (a). They differ in the way the pal-permission structure emerges from the set of axioms. In the first one, the pal-permission structure is such that a player is allowed to cooperate at any activity level if, and only if, its superiors in the hierarchy cooperate at their maximal level. In the second one, the palpermission structure is such that a player is allowed to cooperate any activity level if its superiors participate (they choose their minimal activity level). In the third one, a player can cooperate at some activity level $j$ if, and only if, its superiors cooperate at least at the activity level $j$. In the latter case, it should be noted that even if the permission structure on the player set is a rooted tree, the induced pal-permission structure is not a rooted-tree. These three axiomatic characterizations are inspired from the axiomatic characterization of the Permission value for TU-games with a permission tree structure recently provided by van den Brink et al. 2015. This approach is interesting for several reasons. First, 
it distinguishes a priori a hierarchical structure between the players from a hierarchical structure on the activity levels of each player. Then, the axiomatic study sheds light on how these two types of hierarchical structures are combined into one pal-permission structure on the player-activity level pairs, taking into account the inter and intra players relationships. Finally, quite different pal-permission structures emerge which have natural but different interpretations.

The paper is organized as follows. After dealing with preliminaries on multi-choice games and the Permission value for TU-games with a permission structure in section 2, we present approach (a) in sections 3.1, 3.2 and 3.3. Section 3.1 introduces pal-permission structures. Section 3.2 deals with the set of feasible coalitions and its properties as well as the sovereign and authorizing parts of a coalition. Section 3.3 introduces the Multi-choice permission value. Section 3.4 is devoted to the presentation of approach (b). Section 3.5 introduces the above three allocation rules for multi-choice games with a permission tree structure. Section 4 contains the axiomatic results. Section 5 concludes.

\section{Preliminaries}

\subsection{Multi-choice games}

For each finite set $A$, its cardinality is denoted by $|A|$. Throughout this paper the finite set of players $N=\{1, \ldots, n\}$ is fixed. In a multi-choice game, each player $i \in N$ has a finite set of pairwise distinct activity levels $M_{i}=\left\{0, \ldots, m_{i}\right\}$, totally ordered from the lowest activity level 0 to the greatest activity level $m_{i}$ and where 0 means that player $i$ does not participate or cooperate. A (multi-choice) coalition is an element $s=\left(s_{1}, \ldots, s_{n}\right) \in \mathcal{M}$ where $\mathcal{M}$ stands for the cartesian product $\prod_{i \in N} M_{i}$. In the coalition $s=\left(s_{1}, \ldots, s_{n}\right)$ each player $i \in N$ chooses the activity level $s_{i} \in M_{i}$. The coalition $m=\left(m_{1}, \ldots, m_{n}\right) \in \mathcal{M}$ corresponds to the grand coalition in which each player cooperates at its greatest activity level. The null coalition is the coalition $(0, \ldots, 0)$ where no player participates. The set $\mathcal{M}$ is endowed with the usual binary relation $\geq$ on $\mathbb{R}^{n}$, which induces a (complete) lattice with greatest element $m$ and least element $(0, \ldots, 0)$. For any two coalitions $a$ and $b$ of $\mathcal{M}, a \vee b$ and $a \wedge b$ denote their least upper bound and their greatest lower bound over $\mathcal{M}$, respectively. A multi-choice game on $N$ is a pair $(m, v)$, where $v: \mathcal{M} \longrightarrow \mathbb{R}$ is a characteristic function which assigns to each coalition $s$ the worth $v(s)$ that coalition $s$ can generate, and such that $v(0, \ldots, 0)=0$. A characteristic function $v$ is monotonic if $v(s) \leq v(t)$ whenever $s \leq t$. We denote the class of multi-choice games on $N$ by $\mathcal{G}$.

Remark 1. In case $M_{i}=\{0,1\}$, for each $i \in N,(\mathcal{M}, \geq)$ is isomorphic to $\left(2^{N}, \supseteq\right)$, where $2^{N}$ stands for the set consisting of all subsets of $N$. In such a case, $(m, v)$ is a TU-game. When $(m, v)$ is a TU-game, we use the usual notation $\left(N, v^{\prime}\right)$, where $v^{\prime}$ is the characteristic function $v^{\prime}: 2^{N} \rightarrow \mathbb{R}$, defined for each $E \subseteq N$ as $v^{\prime}(E)=v\left(1_{E}, 0_{N \backslash E}\right)$.

For each $i \in N$, let $M_{i}^{+}=M_{i} \backslash\{0\}$ be player $i$ 's finite set of positive activity levels. Further, define $M^{+}:=\cup_{i \in N}\left(\{i\} \times M_{i}^{+}\right)$as the set of player-activity level pairs. A pair 
$(i, j) \in M^{+}$is a dummy pair if the marginal contribution of any activity level $l$ of player $i$ is null when $l \geq j$. Formally, if $(i, j) \in M^{+}$is dummy, then for each $s_{-i} \in \Pi_{k \in N \backslash i} M_{k}$ and $j \leq l \leq m_{i}$, it holds that

$$
v\left(s_{-i}, j-1\right)=v\left(s_{-i}, l\right)
$$

Observe that if $(i, j)$ is a dummy pair, then it holds that $\left(i, j^{\prime}\right)$ is also a dummy pair for any $j^{\prime} \geq j$. The minimal effort game $\left(m, u_{t}\right) \in \mathcal{G}$, induced by $t \in \mathcal{M}, t \neq(0, \ldots, 0)$, is defined as:

$$
\forall s \in \mathcal{M}, \quad u_{t}(s)= \begin{cases}1 & \text { if } s_{i} \geq t_{i} \text { for each } i \in N \\ 0 & \text { otherwise. }\end{cases}
$$

The collection of minimal effort games $\left\{\left(m, u_{t}\right): t \leq m, t \neq(0, \ldots 0)\right\}$ forms a basis for $\mathcal{G}$ so that each characteristic function $v$ admits a unique linear decomposition in terms of minimal effort games as follows:

$$
v=\sum_{t \leq m} \Delta_{v}(t) u_{t}, \quad \text { where } \quad \Delta_{v}(t)=v(t)-\sum_{s \leq t, s \neq t} \Delta_{v}(s)
$$

where $\Delta_{v}(t)$ is the so-called Harsanyi dividend of coalition $t$ (see Hsiao and Raghavan [1992]). From (1), we immediately get the following lemma.

Lemma 1. If $(i, j)$ is a dummy pair, then $\Delta_{v}(s)=0$ whenever $s_{i} \geq j$.

For any multi-choice game $(m, v) \in \mathcal{G}$, a payoff vector $x \in \mathbb{R}^{\left|M^{+}\right|}$assigns a payoff $x_{i j} \in \mathbb{R}$ to each pair $(i, j) \in M^{+}$. An allocation rule on $\mathcal{G}$ is a map $f$ that assigns a unique payoff vector $f(m, v) \in \mathbb{R}^{\left|M^{+}\right|}$to each $(m, v) \in \mathcal{G}$. For each coalition $s$, we assume that each player $i$ is able to implement any positive activity level $j$ lower than $s_{i}$. The interpretation is that the activity level $s_{i}$ has been reached step by step, and we say that the pair $(i, j)$ is admissible with respect to $s$. For each $s \in \mathcal{M}$ define the set of admissible pairs $B(s)$ as follows:

$$
\forall s \in \mathcal{M}, \quad B(s)=\left\{(i, j) \in M^{+} \mid j \leq s_{i}\right\} .
$$

A well-known allocation rule for multi-choice games is the one introduced by Derks and Peters [1993] and characterized by Klijn et al. [1999]. This rule, known as the DP (DerksPeters) value, distributes the dividend of each coalition $s$ equally among the admissible pairs in $B(s)$. Formally, for each $(m, v) \in \mathcal{G}$, the $D P$ rule is defined as:

$$
\forall(i, j) \in M^{+}, \quad D P_{i j}(m, v)=\sum_{\substack{s \in \mathcal{M} \\(i, j) \in B(s)}} \frac{\Delta_{v}(s)}{|B(s)|},
$$

where $|B(s)|=\sum_{h \in N} s_{h}$ for each $s \in \mathcal{M}$, by definition (2). Observe that for each minimal effort game $\left(m, u_{t}\right)$, the worth $u_{t}(m)=1$ is shared equally among the admissible pairs in $B(t)$ :

$$
\forall(i, j) \in M^{+}, \quad D P_{i j}\left(m, u_{t}\right)= \begin{cases}\frac{1}{|B(t)|} & \text { if }(i, j) \in B(t) \\ 0 & \text { else. }\end{cases}
$$


Remark also that if $(m, v)$ is a TU-game, then $B(s)$ reduces to the set $\left\{i \in N \mid s_{i}>0\right\}$, and the $D P$ value applied to $(m, v)$ coincides with the Shapley value $S h$ of $\left(N, v^{\prime}\right)$, which distributes the dividend of each coalition equally among its members (Shapley [1953]), that is:

$$
\forall i \in N, \quad S h_{i}\left(N, v^{\prime}\right)=\sum_{S \in 2^{N}: S \ni i} \frac{\Delta_{v^{\prime}}(S)}{|S|} .
$$

\subsection{Permission structures on a player set}

In this section, we present permission structures on $N$ and TU-games with a permission structure as introduced in Gilles et al. [1992]. We also introduce the axiomatic characterization proposed in van den Brink et al. [2015] of the (conjunctive) Permission value which is the most popular allocation for TU-games with a permission structure.

In the framework of TU-games, Gilles et al. [1992 consider situations in which some players are part of hierarchical (or permission) structure such that some players need the permission from certain other players to cooperate. Formally, a permission structure defined on the player set $N$ is a map $S: N \longrightarrow 2^{N}$. The relationship $j \in S(i)$ means that $j$ needs the permission from $i$ before cooperating. Equivalently, we also denote $i \in S^{-1}(j)$ if and only if $j \in S(i)$. In this case, player $i$ is the predecessor of $j$ while $j$ is the successor of $i$. The trivial structure $S^{0}$ is such that, for each $i \in N, S^{0}(i)=\emptyset$. The transitive closure of a permission structure $S$ is a permission structure $\hat{S}$ such that, for each $i \in N$, we have $j \in \hat{S}(i)$ if and only if there exists a path $i=h_{1}, h_{2}, \ldots, h_{k}=j$ such that $h_{k} \in S\left(h_{k-1}\right), \ldots, h_{2} \in S\left(h_{1}\right)$. The players in $\hat{S}(i)$ are called the subordinates of $i$ in $S$, and the players in $\hat{S}^{-1}(i):=\{j \in N: i \in \hat{S}(j)\}$ are called the superiors of $i$ in $S$. A cycle occurs in the structure when there exists a path $i=h_{1}, h_{2}, \ldots, h_{k}=i$ such that $h_{k} \in S\left(h_{k-1}\right), \ldots, h_{2} \in S\left(h_{1}\right)$. A permission tree structure is an acyclic permission structure where each player has at most one predecessor and only one player has no predecessor. Such a player is referred to as the root of the tree. A distinction is made between the conjunctive approach and the disjunctive approach. In the conjunctive approach as developed in Gilles et al. [1992] and van den Brink and Gilles [1996], a player needs permission from all its predecessors (if it has any) in the permission structure. On the other hand, in the disjunctive approach as developed in Gilles and Owen [1994] and van den Brink [1997] a player needs permission from at least one of its predecessors (if it has any). Both approaches coincide on permission tree structures since each player has at most one predecessor.

In the class of TU-games with a permission structure, van den Brink and Gilles [1996] introduce an new allocation rule called the (conjunctive) Permission value. Given a TU-game with a permission structure $\left(N, v^{\prime}, S\right)$, the Permission value $\rho$ is computed as the Shapley value of a restricted TU-game $\left(N, R_{S}\left(v^{\prime}\right)\right)$ defined in the following way. The worth $R_{S}\left(v^{\prime}\right)(E)$ of each coalition $E \in 2^{N}$ is the worth $v^{\prime}(\sigma(E))$ generated by the largest, and possibly empty, sub-coalition $\sigma(E) \subseteq E$ containing all the predecessors of each member of $\sigma(E)$. Formally, 
the Permission value is the allocation rule defined as:

$$
\rho\left(N, v^{\prime}, S\right)=\operatorname{Sh}\left(N, R_{S}\right)
$$

Several axiomatic characterizations of the Permission value have been conducted in van den Brink and Gilles [1996], van den Brink et al. [2015], van den Brink et al. [2017], and van den Brink et al. [2018]. In this paper, we build on the axiomatic characterization contained in Theorem 5.6 in van den Brink et al. [2015] for TU-games with a permission tree structure. Consider an allocation rule $f$ for TU-games with a permission tree structure. The first axiom is the standard axiom of efficiency.

Axiom 1 (Efficiency). For each TU-game with a permission tree structure $\left(N, v^{\prime}, S\right)$, it holds that:

$$
\sum_{i \in N} f_{i}\left(N, v^{\prime}, S\right)=v(N)
$$

In a TU-game, if $(i, 1)$ is a dummy pair we just say that $i$ is dummy player (also called a null player), that is, $v^{\prime}(E)-v^{\prime}(E \backslash i)=0$ for each coalition $E$. In a TU-game with a permission tree structure, an inessential player is a dummy player such that each of its subordinates in the permission structure is also a dummy player. The next axiom requires that inessential players should obtain a null payoff.

Axiom 2 (Inessential player property). For each TU-game with a tree permission structure $\left(N, v^{\prime}, S\right)$ and each inessential player $i \in N$, it holds that:

$$
f_{i}\left(N, v^{\prime}, S\right)=0 .
$$

Consider any two players $i, j \in N$ such that $j \in S(i)$. Define the associated game $\left(N, v_{-i j}^{\prime}, S\right)$ where players in $\{j\} \cup \hat{S}(j)$ are not cooperating as follows:

$$
\forall E \in 2^{N} \backslash\{\emptyset\}, \quad v_{-i j}^{\prime}(E)=v(E \backslash(\hat{S}(j) \cup\{j\})) .
$$

In $\left(N, v_{-i j}^{\prime}, S\right)$, all subordinates of $i$ are dummy players. The interpretation is that player $i$ vetoes any coalition that contains $j$ or any of its subordinates but does not contain player $i$. The next axiom states that the resulting changes in payoffs of player $i$ when it is enforcing its power over $j \in S(i)$ and all its subordinates equal the changes in payoff to the player $j \in S(i)$.

Axiom 3 (Permission fairness). For each TU-game with a permission tree structure $\left(N, v^{\prime}, S\right)$ and each pair of distinct players $\{i, j\} \subseteq N$ such that $j \in S(i)$, it holds that:

$$
f_{i}\left(N, v^{\prime}, S\right)-f_{i}\left(N, v_{-i j}^{\prime}, S\right)=f_{j}\left(N, v^{\prime}, S\right)-f_{j}\left(N, v_{-i j}^{\prime}, S\right) .
$$

Theorem 1. van den Brink et al. [2015], Theorem 5.6) An allocation rule for TU-games with a permission tree structure satisfies Efficiency, Inessential player property and Permission fairness if and only if it is the Permission value.

For more details on TU-games with a permission structure, their allocation rules, and the possible applications in economics and operations research, we refer to van den Brink 2017. 


\section{$3 \quad$ Two approaches}

In this section we elaborate on the two approaches (a) and (b) presented in the Introduction. Sections 3.1 - 3.3 concern approach (a). Section 3.4 presents approach (b) and section 3.5 introduces three new allocation rules for multi-choice games with a permission tree structure.

\subsection{Pal-permission structures}

In the context of multi-choice games, we consider situations in which some players cannot cooperate at a certain activity level until some other players have reached a certain activity level. To model such situations, we introduce player-activity level permission structures (pal-permission structures henceforth) which extend permission structures from $N$ to $M^{+}$.

Definition 1. Consider any nonempty set of player-activity level pairs $M^{+}$. A pal-permission structure on $M^{+}$is a mapping $P: M^{+} \longrightarrow 2^{M^{+}}$such that the relationship $(k, l) \in P(i, j)$ indicates that player $k$ cannot cooperate at activity level $l$ until $i$ has reached its activity level $j$. Thus the pair $(k, l) \in M^{+}$is a successor of the pair $(i, j) \in M^{+}$. And, as for the case of permission structures on $N$ the notation $(i, j) \in P^{-1}(k, l)$ means that the pair $(i, j)$ is a predecessor of the pair $(k, l)$. The mapping $P$ is assumed to be asymmetric, that is,

$$
\forall i \in N, \forall(i, j) \in M_{i}^{+}, \quad(i, j) \notin P(i, j) .
$$

The notation $\hat{P}$ stands for the transitive closure of $P$, the set of superiors and subordinates of a player are defined accordingly.

Remark 2. Because player's $i$ set of activity levels $M_{i}$ is totally ordered from the null activity level 0 to the greatest activity level $m_{i}$, the set of pairs $\{i\} \times M_{i}^{+}$can be also totally ordered as follows: $(i, 1)<(i, 2)<\ldots<\left(i, m_{i}\right)$. From this point of view, we obtain a pal-permission structure between $i$ 's activity levels.

The trivial pal-permission structure $P^{0}$ on $M^{+}$is the pal-permission structure consistent with the pal-permission structure on $\{i\} \times M_{i}^{+}$as defined in Remark 2 and containing no other relation. Therefore, in $P^{0}$, a player cannot cooperate at its activity level $j$ if it has not reached its activity level $j-1$ and no pair formed by two player-activity level pairs involving distinct players are in relation. Formally, $P^{0}: M^{+} \longrightarrow 2^{M^{+}}$is defined as:

$$
\forall i \in N, \forall j \in M_{i}^{+} \backslash\left\{m_{i}\right\}, \quad(i, j+1) \in P^{0}(i, j) .
$$

Remark 3. In the same way as a multi-choice game with the trivial permission structure $\left(m, v, S^{0}\right)$ can be assimilated to the multi-choice game $(m, v)$, a multi-choice game augmented with the trivial pal-permission structure $\left(m, v, P^{0}\right)$ can be seen as the multi-choice game $(m, v)$. 
Consider any two pal-permission structures $P$ and $P^{\prime}$. We say that $P$ extends $P^{\prime}$ if the following holds:

$$
\forall(i, j),(k, l) \in M^{+}, \quad\left[(i, j) \in P^{\prime}(k, l)\right] \Longrightarrow[(i, j) \in P(k, l)]
$$

Finally, we say that a pal-permission structure $P$ is consistent with respect to $P^{0}$ or simply consistent if it extends $P^{0}$. Thus, in a consistent pal-permission structure, a player cannot cooperate at its activity level $j$ if it has not reached its activity level $j-1$.

Example 1. Consider the player set $N=\{F, W\}$ and the vector of maximal activity levels $m=(3,2)$ where $m_{F}=3$ and $m_{W}=2$. Consider the pal-permission structures $P^{0}$ and $P$, such that $P$ extends $P^{0}$. We represent the two pal-permission structures by the following digraphs:

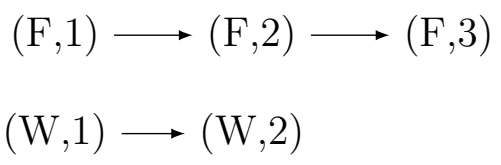

(a) $P^{0}$

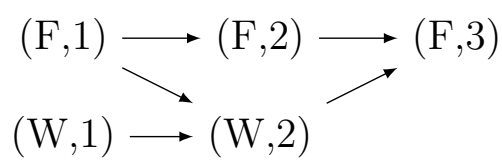

(b) $P$

In $P^{0}, F$ needs to achieve its activity levels 1 and 2 before deploying its activity level 3. In P, F not only has to achieve its activity levels 1 and 2 to deploy its activity level 3, but also needs that $W$ achieves its activity levels 1 and 2.

In the following, we only consider the set of consistent and acyclic pal-permission structures that we denote by $\mathcal{A}^{+}$.

\subsection{Feasible multi-choice coalitions}

A pal-permission structure $P \in \mathcal{A}^{+}$influences the possibilities of cooperation in a multichoice game in the following way. Let $(k, l)$ be a predecessor of $(i, j)$ with respect to $P$ and $s$ be a coalition such that $s_{i}=j$. In order for player $i$ to be able to to cooperate at activity level $j$, player $k$ must have an activity level of at least $l$ in $s$, that is, $s_{k} \geq l$. In this sense, the player-activity level pair $(i, j)$ needs the permission of the player-activity level pair $(k, l)$ to cooperate in $s$. A coalition $s$ is feasible if each player-activity level pair has the permission of all its superiors in $s$. This way of introducing the influence of the permission relationships in a coalition is a natural generalization of the conjunctive approach from TU-games to multi-choice games.

Definition 2. Consider a pal-permission structure $P \in \mathcal{A}^{+}$on $M^{+}$. The set of feasible coalitions $\mathcal{F}(m, P)$ is defined as:

$$
\left.\mathcal{F}(m, P)=\left\{s \in \mathcal{M}: s_{i}>0 \Longrightarrow \forall(k, l) \in \hat{P}^{-1}\left(i, s_{i}\right), s_{k} \geq l\right)\right\}
$$

To fix the ideas, let us consider the following example. 
Example 2. Consider again Example 1 where the pal-permission structure $P$ is given in Figure (b). A coalition $s$ is of the form $\left(s_{F}, s_{W}\right)$, where $s_{F} \in\{0,1,3\}$ is the activity level of $F$ and $s_{W} \in\{0,1,2\}$ the activity level of $W$. Then, the set of feasible coalitions is defined as:

$$
\mathcal{F}(m, P)=\mathcal{M} \backslash\{(0,2),(3,0),(3,1)\} .
$$

For instance, consider the coalition $\left(s_{F}, s_{W}\right)=(0,2)$. This coalition is not feasible because of the player-activity level pair $(F, 0) \in \hat{P}^{-1}(W, 2)$. This means that $W$ needs that $F$ plays an activity level $s_{F} \geq 1$ to cooperate at level 2 , which is not the case in coalition $(0,2)$. On the other hand, coalition $\left(s_{F}, s_{W}\right)=(0,1)$ is feasible because $W$ does not need the permission of any activity level of $F$ to to be able to cooperate at activity level 1.

The next lemma establishes that the set of feasible coalitions forms a (finite) lattice whose greatest element is the grand coalition and the least element is the null coalition.

Lemma 2. Consider any $(m, v, P) \in \mathcal{G}^{\mathcal{A}^{+}}$. The set $\mathcal{F}(m, P)$ satisfies the following properties:

(i) $(0, \ldots, 0) \in \mathcal{F}(m, P)$;

(ii) $m \in \mathcal{F}(m, P)$;

(iii) for each $s, t \in \mathcal{F}(m, P)$, it holds that $s \wedge t$ and $s \vee t$ are still elements of $\mathcal{F}(m, P)$.

Proof. Consider any $(m, v, P) \in \mathcal{G}^{\mathcal{A}^{+}}$.

(i) Consider the null coalition $s=(0, \ldots, 0)$. Because there is no $i \in N$ such that $s_{i}>0$, by definition of $\mathcal{F}(m, P)$ conclude that $(0, \ldots, 0) \in \mathcal{F}(m, P)$.

(ii) Consider the grand coalition $m$. Because each coordinate of $m$ is maximal, by definition of $\mathcal{F}(m, P)$ conclude that $m \in \mathcal{F}(m, P)$.

(iii) Consider two feasible coalitions $s, t \in \mathcal{F}(m, P)$ and set $a=s \wedge t$ and $b=s \vee t$. To show: $a, b \in \mathcal{F}(m, P)$.

First, we show that $a \in \mathcal{F}(m, P)$. We proceed by contradiction. Suppose that $a \notin$ $\mathcal{F}(m, P)$. By definition, there exist $i, k \in N$ such that $a_{i}>0, a_{k}<l$, and $(k, l) \in$ $\hat{P}^{-1}\left(i, a_{i}\right)$. Observe that for such a player $i$, it holds that either $a_{i}=s_{i}$ or $a_{i}=t_{i}$. Without loss of generality, suppose that $a_{i}=s_{i} \leq t_{i}$. In the same way, either $a_{k}=s_{k}$ or $a_{k}=t_{k}$.

- If $a_{k}=s_{k}$, then $s_{k}<l$. Because $a_{i}=s_{i}$, it follows that $(k, l) \in \hat{P}^{-1}\left(i, s_{i}\right)$ and so $s \notin \mathcal{F}(m, P)$, a contradiction.

- If $a_{k}=t_{k}$, then $t_{k}<l$. Because $a_{i}=s_{i} \leq t_{i}$ and $(k, l) \in \hat{P}^{-1}\left(i, s_{i}\right)$, by consistency of $P$ we also have $(k, l) \in \hat{P}^{-1}\left(i, t_{i}\right)$. Thus, we have $t \notin \mathcal{F}(m, P)$, a contradiction.

All in all, we have shown that $a \in \mathcal{F}(m, P)$.

The proof that $b \in \mathcal{F}(m, P)$ is similar and so is left to the reader. 
Because $\mathcal{F}(m, P)$ is a finite sub-lattice of $\mathcal{M}$, for each coalition $s$, we can define the supremum of the feasible coalitions smaller than $s$ and the infimum of the feasible coalitions larger than $s$. In the first case, we obtain the greatest feasible coalition $\sigma(s) \in \mathcal{F}(m, P)$ such that $\sigma(s) \leq s$; in the second case, we obtain the smallest feasible coalition $\alpha(s) \in \mathcal{F}(m, P)$ such that $\alpha(s) \geq s_{i}$ for each $i \in N$. To paraphrase Gilles et al. 1992, $\sigma(s)$ is the sovereign part of $s$ and $\alpha(s)$ is the authorizing part of $s$.

Definition 3. Consider any pal-permission structure $P$ on $M^{+}$and any multi-choice coalition $s \in \mathcal{M}$. The sovereign and authorizing parts of $s$ with respect to $P$ are respectively given by:

$$
\sigma(s)=\bigvee_{\substack{t \in \mathcal{F}(m, P) \\ t \leq s}} t, \quad \alpha(s)=\bigwedge_{\substack{t \in \mathcal{F}(m, P) \\ t \geq s}} t
$$

Remark 4. Obviously, for each $t \in \mathcal{F}(m, P)$, it holds that $\sigma(t)=\alpha(t)=t$; in particular, $\sigma(m)=\alpha(m)=m$. Finally, for any $s, t \in \mathcal{M}$ such that $s \leq t$, it holds that $\sigma(s) \leq \sigma(t)$ and $\alpha(s) \leq \alpha(t)$.

To close this section, the following lemma provides interesting properties of the sovereign and authorizing parts of a multi-choice coalition. This lemma echoes Proposition 3.5 in Gilles et al. 1992 for coalitions of players.

Lemma 3. Consider any pal-permission structure $P$ on $M^{+}$and any pair coalitions $s, t \in$ $\mathcal{M}$. It holds that:

(i) $\sigma(s) \vee \sigma(t) \leq \sigma(s \vee t)$;

(ii) $\sigma(s) \wedge \sigma(t)=\sigma(s \wedge t)$;

(iii) $\alpha(s) \vee \alpha(t)=\alpha(s \vee t)$;

(iv) $\alpha(s \wedge t) \leq \alpha(s) \wedge \alpha(t)$.

Proof. Consider any pal-permission structure $P$ on $M^{+}$and any pair of multi-choice coalitions $s, t \in \mathcal{M}$.

(i) By definition, $s \leq s \vee t$ and $t \leq s \vee t$ and so $\sigma(s) \leq \sigma(s \vee t)$ and $\sigma(t) \leq \sigma(s \vee t)$ (see Remark 4). By definition of the supremum,

$$
\sigma(s) \vee \sigma(t) \leq \sigma(s \vee t) \vee \sigma(s \vee t)=\sigma(s \vee t)
$$


(ii) By definition, $t \geq s \wedge t$ and $s \geq s \wedge t$ and so $\sigma(s) \geq \sigma(s \wedge t)$ and $\sigma(t) \geq \sigma(s \wedge t)$. This implies that $\sigma(s) \wedge \sigma(t) \geq \sigma(s \wedge t)$. On the other hand, by definition of $\sigma, \sigma(s) \leq s$ and $\sigma(t) \leq t$. Thus, we have:

$$
\begin{aligned}
& \sigma(s) \wedge \sigma(t) \leq s \wedge t \\
\Longleftrightarrow & \sigma(\sigma(s) \wedge \sigma(t)) \leq \sigma(s \wedge t) \\
\Longleftrightarrow & \sigma(s) \wedge \sigma(t) \leq \sigma(s \wedge t),
\end{aligned}
$$

where the equality

$$
\sigma(\sigma(s) \wedge \sigma(t))=\sigma(s) \wedge \sigma(t)
$$

comes from the fact that $\sigma(s) \wedge \sigma(t) \in \mathcal{F}(m, P)$ by Lemma 2. Conclude that $\sigma(s) \wedge$ $\sigma(t)=\sigma(s \wedge t)$.

(iii) By definition of $\alpha, \alpha(s) \geq s$ and $\alpha(t) \geq t$, from which we get:

$$
\begin{aligned}
& \alpha(s) \vee \alpha(t) \geq s \vee t \\
\Longleftrightarrow & \alpha(\alpha(s) \vee \alpha(t)) \geq \alpha(s \vee t) \\
\Longleftrightarrow & \alpha(s) \vee \alpha(t) \geq \alpha(s \vee t)
\end{aligned}
$$

On the other hand, we have $s \leq s \vee t$, which implies that $\alpha(s) \leq \alpha(s \vee t)$. By definition we have $\alpha(t) \leq \alpha(s \vee t)$. It implies that $\alpha(t) \vee \alpha(s) \leq \alpha(s \vee t)$. From the above arguments, we arrive at $\alpha(s) \vee \alpha(t)=\alpha(s \vee t)$.

(iv) The fact that $s \wedge t \leq s$ and $s \wedge t \leq t$ implies that $\alpha(s \wedge t) \leq \alpha(s)$ and $\alpha(s \wedge t) \leq \alpha(t)$, and so $\alpha(s \wedge t) \leq \alpha(s) \wedge \alpha(t)$.

\subsection{Allocation rules for multi-choice games with a pal-permission structure}

A multi-choice game $(m, v) \in \mathcal{G}$ augmented with a pal-permission structure $P \in \mathcal{A}^{+}$is the triple $(m, v, P)$. The class of multi-choice games with a pal-permission structure is denoted by $\mathcal{G}^{\mathcal{A}^{+}}$. An allocation rule on $\mathcal{G}^{\mathcal{A}^{+}}$is a map $f$ that assigns a unique payoff vector $f(m, v, P) \in \mathbb{R}^{|M+|}$ to each $(m, v, P) \in \mathcal{G}^{\mathcal{A}^{+}}$.

In a multi-choice game with a pal-permission structure $(m, v, P)$, the multi-choice game $(m, v)$ describes the possibilities of cooperation regardless of the restrictions induced by the pal-permission structure. To take these restrictions into account, a new multi-choice game $\left(m, \mathcal{R}_{P}(v)\right) \in \mathcal{G}$ is constructed. This game takes into account both the cooperation possibilities represented by $(m, v)$ and the permission relationships represented by $P$. The resulting multi-choice game $\left(m, \mathcal{R}_{P}(v)\right)$ is the (conjunctive) restriction of the multi-choice game of $(m, v)$ induced by the pal-permission structure $P$. Then, the Derks-Peters value $D P$ is applied to $\left(m, \mathcal{R}_{P}(v)\right)$. This allows to define an allocation rule for multi-choice games with 
a pal-permission structure. Because the $D P$ value is a generalization of the Shapley value, and the Permission value is the Shapley value of a TU-games restricted by a permission structure, it follows that the Derks-Peters value $D P$ applied to $\left(m, \mathcal{R}_{P}(v)\right)$ can be viewed as a possible generalization of the Permission value from TU-games with a permission structure to multi-choice games with a pal-permission structure.

Definition 4. Consider any $(m, v, P) \in \mathcal{G}^{\mathcal{A}^{+}}$. The restriction of $(m, v)$ on $P$ is the multichoice game $\left(m, \mathcal{R}_{P}(v)\right)$ where the characteristic function $\mathcal{R}_{P}(v)$ is defined as:

$$
\mathcal{R}_{P}(v)=v \circ \sigma
$$

Thus, the worth $\mathcal{R}_{P}(v)(s)$ of the multi-choice coalition $s$ is the worth $v(\sigma(s))$ of its sovereign part. In other words, the mapping $\sigma$ indicates how $s$ is evaluated: the sovereign part with respect to $P$, that is the greatest feasible coalition smaller than $s$, generates a worth $v(\sigma(s))$ when $s$ forms.

Remark 5. If $(m, v)$ is monotonic, then $\left(m, \mathcal{R}_{P}(v)\right)$ is also monotonic. Indeed, if $s \leq t$ implies $v(s) \leq v(t)$ we have $\sigma(s) \leq \sigma(t)$ and so $\mathcal{R}_{P}(v)(s) \leq \mathcal{R}_{P}(v)(t)$. Fix the set of multichoice coalitions $\mathcal{M}$. The operator $\mathcal{R}_{P}$ is linear on the characteristic fonctions on $\mathcal{M}$, that is, $\mathcal{R}_{P}(v+\gamma w)=\mathcal{R}_{P}(v)+\gamma \mathcal{R}_{P}(w)$ for each $v$, w on $\mathcal{M}$ and each $\gamma \in \mathbb{R}$.

Lemma 4. Consider any $\left(m, u_{s}, P\right) \in \mathcal{G}^{\mathcal{A}^{+}}, s \in \mathcal{M}$ such that $s \neq(0, \ldots, 0)$. It holds that:

$$
\mathcal{R}_{P}\left(u_{s}\right)=u_{\alpha(s)}
$$

Proof. Consider any $\left(m, u_{s}, P\right) \in \mathcal{G}^{\mathcal{A}^{+}}$and any $s \in \mathcal{M}$ such that $s \neq(0, \ldots, 0)$. By definition of $\mathcal{R}_{P}\left(u_{s}\right)$,

$$
\forall t \in \mathcal{M}, \quad \mathcal{R}_{P}\left(u_{s}\right)(t)=u_{s}(\sigma(t))= \begin{cases}1 & \text { if } \sigma(t) \geq s \\ 0 & \text { else }\end{cases}
$$

First, let us show the following equivalence:

$$
\sigma(t) \geq s \Longleftrightarrow t \geq \alpha(s)
$$

- Suppose that $\sigma(t) \geq s$. We have $\alpha(\sigma(t))=\sigma(t) \geq \alpha(s)$. Because $t \geq \sigma(t)$, we obtain $t \geq \alpha(s)$

- Reciprocally, if $t \geq \alpha(s)$, then $\sigma(t) \geq \sigma(\alpha(s))=\alpha(s) \geq s$.

Thus, we obtain that:

$$
\forall t \in \mathcal{M}, \quad \mathcal{R}_{P}\left(u_{s}\right)(t)= \begin{cases}1 & \text { if } t \geq \alpha(s), \\ 0 & \text { else }\end{cases}
$$

so that $\mathcal{R}_{P}\left(u_{s}\right)=u_{\alpha(s)}$, as desired. 
By Lemma 4 and the linearity of the operator $\mathcal{R}_{P}$, the characteristic function $\mathcal{R}_{P}(v)$ can we rewritten as:

$$
\mathcal{R}_{P}(v)=\mathcal{R}_{P}\left(\sum_{s \in \mathcal{M}} \Delta_{v}(s) u_{s}\right)=\sum_{s \in \mathcal{M}} \Delta_{v}(s) \mathcal{R}_{P}\left(u_{s}\right)=\sum_{s \in \mathcal{M}} \Delta_{v}(s) u_{\alpha(s)} .
$$

We introduce a new allocation rule on multi-choice games with a pal permission structure, called the Multi-choice permission value, which allocates the worth of the grand coalition according to the Derks-Peters value applied to the restricted game induced by the palpermission structure.

Definition 5. The Multi-choice permission value $\Upsilon$ on $\mathcal{G}^{\mathcal{A}^{+}}$is defined as:

$$
\forall(i, j) \in M^{+}, \quad \Upsilon_{i j}(m, v, P)=D P_{i j}\left(m, \mathcal{R}_{P}(v)\right) .
$$

From (1), (4), and the fact that the DP value is linear (see Klijn et al. [1999]), $\Upsilon$ can be rewritten as follows:

$$
\forall(i, j) \in M^{+}, \quad \Upsilon_{i j}(m, v, P)=\sum_{\substack{s \in \mathcal{M} \\(i, j) \in B(\alpha(s))}} \frac{\Delta_{v}(s)}{|B(\alpha(s))|}=\sum_{s \in \mathcal{M}: j \leq \alpha_{i}(s)} \frac{\Delta_{v}(s)}{\sum_{h \in N} \alpha_{h}(s)} .
$$

The Multi-choice permission value $\Upsilon$ distributes the dividend of each coalition $s$ equally among the admissible pairs of the authorizing part $\alpha(s)$ of $s$. It follows that $\Upsilon$ is a generalization of both the DP value and the Permission value $\rho$. Indeed, in case $(m, v)$ is a TU-game, i.e., $m=(1, \ldots, 1)$, the Multi-choice Permission value coincides with the Permission value

as in (5). In case $P=P^{0}$, the Multi-choice permission value coincides with the $D P$ value as in (3).

\subsection{Pal-permission structures induced by a permission tree struc- ture}

Consider the situation where the players are part of a hierarchical organization and the sets of activity levels are totally ordered from the lowest activity level to the greatest activity level. In such a situation, several pal-permission structures can be created from the permission structure on the player set and the sets of activity levels. This amounts to say that the relations between the players, the inter-individual relationships, and the relations between the activity levels of each player, the intra-individual relationships, are a priori two independent objects. This means that they can be aggregated to obtain a single hierarchical organization represented by a pal-permission structure. This leads to the following definition.

Definition 6. A pal-permission structure induced by a permission tree structure $S \in \mathcal{T}$ and the vector of maximal activity levels $m$ is a consistent and acyclic pal-permission structure $S^{+} \in \mathcal{A}^{+}$constructed from the hierarchical relationships determined by $S$ and the set of the activity levels represented by $m$. 
We focus the study to three particular induced pal-permission structures that we detail below. For a permission tree structure $S \in \mathcal{T}$ and $m$,

- the m-permission structure on $M^{+}$induced by $S$ and $m$, is the consistent and acyclic pal-permission structure in which a player can cooperate only if all its superiors in $S$ participate by choising their maximal activity level. The resulting structure is denoted by $S_{m}^{+}$. Formally, $S_{m}^{+} \in \mathcal{A}^{+}$is such that:

$$
\forall i, j \in N, \quad[j \in S(i)] \Longleftrightarrow\left[(j, 1) \in S_{m}^{+}\left(i, m_{i}\right)\right]
$$

- The 1-permission structure on $M^{+}$induced by $S$ and $m$ is the consistent and acyclic pal-permission structure in which a player can cooperate only if all its superiors in $S$ participate. Such a structure is denoted by $S_{1}^{+}$. Formally, $S_{1}^{+} \in \mathcal{A}^{+}$is such that:

$$
\forall i, j \in N, \quad[j \in S(i)] \Longleftrightarrow\left[(j, 1) \in S_{1}^{+}(i, 1)\right]
$$

- the Full-permission structure on $M^{+}$induced by $S$ and $m$ is the consistent and acyclic pal-permission structure in which a player can cooperate at a certain activity level $l$ if its superiors in $S$ participates at level $l$ or more. We assume that, for each $j \in S(i), m_{j} \leq m_{i}$. This assumption leads to a specific class of games which will be detailed later (in Section 4.3). Formally, $S_{F}^{+} \in \mathcal{A}^{+}$is such that:

$$
\forall i, j \in N, \forall l \in M_{j}, \quad[j \in S(i)] \Longleftrightarrow\left[(j, l) \in S_{F}^{+}(i, l)\right]
$$

Observe that, for each $S \in \mathcal{T}$ and each $m$, the structures $S_{m}^{+}, S_{1}^{+}$and $S_{F}^{+}$are uniquely determined and belong to the consistent and acyclic pal-permission structures $\mathcal{A}^{+}$by construction.

Example 3. Consider once again Example 1, where the two players are $\{F, W\}$ and $m=$ $(3,2)$. Consider the permission tree structure $S \in \mathcal{T}$ such that $W \in S(F)$. The induced palpermission structures $S_{m}^{+}, S_{1}^{+}$and $S_{F}^{+}$are represented by figures $(a),(b)$ and $(c)$, respectively.

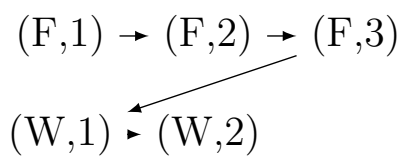

(a) m-permission structure $S_{m}^{+}$

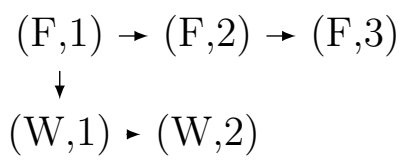

(b) 1-permission structure $S_{1}^{+}$

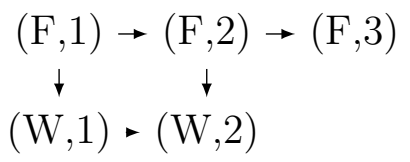

(c) Full-permission structure $S_{F}^{+}$

Although $S$ is a permission tree structure, $S^{+}$is not necessarily a pal-permission tree structure (see $S_{F}^{+}$in the above example). 


\subsection{Three new allocation rules for multi-choice games with a per- mission structure}

Let $\mathcal{G}^{\mathcal{T}}$ be the class of games $(m, v, S)$ where the players in $N$ play the multi-choice game $(m, v) \in \mathcal{G}$ and are hierarchially organized according to the permission tree structure $S \in \mathcal{T}$ on $N$. We consider three possible allocation rules on $\mathcal{G}^{\mathcal{T}}$. Each of these allocation rules are determined by the Multi-choice permission value $\Upsilon$ for multi-choice games with a pal permission structure. The first one consists in applying the Multi-choice permission value on the multi-choice game with the m-permission structure. The second one consists in applying the Multi-choice permission value on the multi-choice game with the 1-permission structure. And the last one consists in applying the Multi-choice permission value on the multi-choice game with the Full-permission structure. Proceeding in this way, the hierarchical interindividual relationships represented by $S$ and the hierarchical intra-individual relationships represented by $m$ are aggregated into a single hierarchical structure represented by a palpermission structure $\left(S_{m}^{+}, S_{1}^{+}\right.$, or $\left.S_{F}^{+}\right)$on $\mathcal{A}^{+}$. Then, the distribution of the payoffs are given by the Derks-Peters value of the restricted game induced by the corresponding pal-permission structure. This leads to the following definitions.

The allocation rule $f^{(+, m)}$ on $\mathcal{G}^{\mathcal{T}}$ is such that:

$$
f^{(+, m)}(m, v, S)=\Upsilon\left(m, v, S_{m}^{+}\right)=D P\left(m, \mathcal{R}_{S_{m}^{+}}(v)\right)
$$

The allocation rule $f^{(+, 1)}$ on $\mathcal{G}^{\mathcal{T}}$ is such that:

$$
f^{(+, 1)}(m, v, S)=\Upsilon\left(m, v, S_{1}^{+}\right)=D P\left(m, \mathcal{R}_{S_{1}^{+}}(v)\right) .
$$

The allocation rule $f^{(+, F)}$ on $\mathcal{G}^{\mathcal{T}}$ is such that:

$$
f^{(+, F)}(m, v, S)=\Upsilon\left(m, v, S_{F}^{+}\right)=D P\left(m, \mathcal{R}_{S_{F}^{+}}(v)\right) .
$$

Example 4. Consider the permission structure of Example 3. For the sake of simplicity, assume that $m_{F}=2$ and $m_{W}=1$ so that $m=(2,1)$. The induced pal-permission structures $S_{m}^{+}, S_{1}^{+}$and $S_{F}^{+}$are represented by figures $(a),(b)$ and $(c)$, respectively.

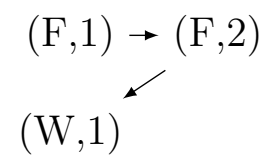

(a) m-permission structure $S_{m}^{+}$

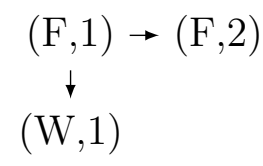

(b) 1-permission structure $S_{1}^{+}$

$$
(\mathrm{F}, 1) \rightarrow(\mathrm{F}, 2)
$$

$(\mathrm{W}, 1)$

(c) Full-permission structure $S_{F}^{+}$

The multi-choice game $v$ is given by the following table:

\begin{tabular}{|c|cccccc|}
\hline$\left(s_{F}, s_{W}\right)$ & $(0,0)$ & $(1,0)$ & $(2,0)$ & $(0,1)$ & $(1,1)$ & $(2,1)$ \\
\hline$v(s)$ & 0 & 1 & 4 & 1 & 3 & 5 \\
\hline
\end{tabular}

The three restricted games corresponding to each induced pal-permission structure are as follows: 


\begin{tabular}{|c|cccccc|}
\hline$\left(s_{F}, s_{W}\right)$ & $(0,0)$ & $(1,0)$ & $(2,0)$ & $(0,1)$ & $(1,1)$ & $(2,1)$ \\
\hline $\mathcal{R}_{S_{m}^{+}}(v)(s)$ & 0 & 1 & 4 & 0 & 1 & 5 \\
\hline $\mathcal{R}_{S_{1}^{+}}(v)(s)$ & 0 & 1 & 4 & 0 & 3 & 5 \\
\hline $\mathcal{R}_{S_{F}^{+}}(v)(s)$ & 0 & 1 & 4 & 0 & 3 & 5 \\
\hline
\end{tabular}

The worth $\mathcal{R}_{S_{m}^{+}}(v)(1,1)=v(1,0)=1$ because $W$ is unable to achieve activity level 1 if $F$ has not reached activity level 2. It follows that $\sigma(1,1)=(1,0)$ under the m-permission structure. Thus, we have that $\mathcal{R}_{S_{m}^{+}}(v)(1,1)=v(\sigma(1,1))=v(1,0)=1$. In a similar way, $\mathcal{R}_{S_{1}^{+}}(v)(0,1)=0$ because $W$ does not have the permission of $F$ to cooperate at level 1 if $F$ is inactive. It follows that $\sigma(0,1)=(0,0)$ under the 1-permission structure. Thus, we have that $\mathcal{R}_{S_{1}^{+}}(v)(0,1)=v(\sigma(0,1))=v(0,0)=0$. In this example, $\mathcal{R}_{S_{1}^{+}}(v)$ and $\mathcal{R}_{S_{F}^{+}}(v)$ coincide because $S_{1}^{+}$and $S_{F}^{+}$coincide in this particular case. Finally, we compute $f^{(+, m)}, f^{(+, 1)}, f^{(+, F)}$ and the DP value.

\begin{tabular}{|c|ccc|}
\hline$(i, j)$ & $(F, 1)$ & $(F, 2)$ & $(W, 1)$ \\
\hline$f^{(+, m)}(m, v, S)$ & 3.17 & 1.17 & 0.67 \\
\hline$f^{(+, 1)}(m, v, S)$ & 2.83 & 1.83 & 0.33 \\
\hline$f^{(+, F)}(m, v, S)$ & 2.83 & 1.83 & 0.33 \\
\hline$D P(m, v)$ & 2.67 & 1.17 & 1.17 \\
\hline
\end{tabular}

As expected $f^{(+, m)}, f^{(+, 1)}$ and $f^{(+, F)}$ allocate a greater payoff to the root $(F, 1)$ than the DP value.

\section{Characterizations results}

This section provides comparable axiomatic characterizations of $f^{(+, m)}, f^{(+, 1)}$, and $f^{(+, F)}$. These axiomatic results preserve the principles of efficiency, permission fairness, and the inessential player contained in Theorem 1 for TU-games with a permission tree structure. However, four types of modifications need to be incorporated due to the multi-choice nature of the game and the two types of hierarchical relationships:

- two, rather than one, principles of permission fairness are introduced. The first one concerns hierarchical inter-individual relationships, the second one concerns intra-individual relationships;

- the above two principles of permission fairness no longer concern the players but the player-activity level pairs;

- the notion of inessential player must be extended to that of inessential player-activity level pair;

- because the allocation rules $f^{(+, m)}, f^{(+, 1)}$ and $f^{(+, F)}$ are built on different pal-permission structures, $S_{m}^{+}, S_{1}^{+}$and $S_{F}^{+}$respectively, each of them satisfies different axioms of permission fairness and inessential player-activity level pair property. 


\subsection{Characterization of $f^{(+, m)}$}

The first axiom is the classical axiom of efficiency.

Axiom 4 (Efficiency). For each $(m, v, S) \in \mathcal{G}^{\mathcal{T}}$, it holds that:

$$
\sum_{i \in N} \sum_{j \in M_{i}^{+}} f_{i j}(m, v, S)=v(m) .
$$

Suppose that a pair $(i, j) \in M^{+}$is dummy in a game $(m, v, S) \in \mathcal{G}^{\mathcal{T}}$. Assume first that $S$ coincides with the trivial permission structure $S^{0}$. Then, player $i$ does not need anyone's permission to participate at level $j$, and the principle incorporated in the dummy level property for multi-choice games applies. This principle indicates that $(i, j)$ gets a null payoff whenever it is a dummy pair. The dummy level property for multi-choice games has been introduced by Klijn et al. [1999 to characterize the $D P$ value and generalizes the null player property for TU-games. But, if $S$ differs from the trivial structure $S^{0}$, although the pair $(i, j)$ is a dummy pair, it might be the case that other players need $(i, j)$ 's permission to fully cooperate. In that case, it seems no longer reasonable to assume that the pair $(i, j)$ gets a zero payoff. The dummy level property may however still be applicable for the case in which $(i, j)$ is a dummy pair and all pairs $(k, l)$ are dummy pairs in $v$, where $k$ is a subordinate of $i$. Formally, a pair $(i, j) \in M^{+}$is a m-inessential pair if it is a dummy pair in $v$ and if, for each of its subordinates $k \in \hat{S}(i)$, it holds that $(k, 1)$ is a dummy pair in $(m, v, S)$. The set of all m-inessential pairs in $(m, v, S)$ is denoted by $I L^{m}(m, v, S)$. The following axiom indicates that each m-inessential pair gets a null payoff.

Axiom 5 (m-inessential pair property). For each $(m, v, S) \in \mathcal{G}^{\mathcal{T}}$, if $(i, j) \in I L^{m}(m, v, S)$, then it holds that:

$$
f_{i j}(m, v, S)=0 .
$$

The following two axioms apply the principle of permission fairness into two directions. Before introducing these axioms, we need a definition. Consider any $(m, v, S) \in \mathcal{G}^{\mathcal{T}}$. For each pair $\left(i, j^{\prime}\right) \in M^{+}$, define the associated $\left(i, j^{\prime}\right)$-game $\left(m, v_{m}^{-\left(i, j^{\prime}\right)}, S\right) \in \mathcal{G}^{\mathcal{T}}$ where in $v_{m}^{-\left(i, j^{\prime}\right)}$, player $i$ stops any activity from level $j^{\prime}$ onwards, and its subordinates are no longer active. For each $s \in \mathcal{M}$, the worth $v_{m}^{-\left(i, j^{\prime}\right)}(s)$ is defined as:

$$
v_{m}^{-\left(i, j^{\prime}\right)}(s)=v(\bar{s}), \quad \text { where } \bar{s}_{k}= \begin{cases}j^{\prime}-1 & \text { if } k=i \text { and } s_{k} \geq j^{\prime} \\ 0 & \text { if } k \in \hat{S}(i), \\ s_{k} & \text { else. }\end{cases}
$$

By construction, for each $k \in \hat{S}(i)$ and each $l \in M_{k}^{+},(k, l) \in I L^{m}\left(m, v_{m}^{-\left(i, j^{\prime}\right)}, S\right)$. Similarly, for each $j \leq j^{\prime} \leq m_{i},\left(i, j^{\prime}\right) \in I L^{m}\left(m, v_{m}^{-\left(i, j^{\prime}\right)}, S\right)$. The first axiom of permission fairness indicates that the changes in payoff of the pairs $(i, j) \in M_{i}^{+}$, for $j \leq j^{\prime}$ are equal.

Axiom 6 (Intra m-fairness). For each $(m, v, S) \in \mathcal{G}^{\mathcal{T}}$ and each $(i, j),\left(i, j^{\prime}\right) \in M^{+}$such that $j^{\prime} \geq j$, it holds that:

$$
f_{i j}(m, v, S)-f_{i j}\left(m, v_{m}^{-\left(i, j^{\prime}\right)}, S\right)=f_{i j^{\prime}}(m, v, S)-f_{i j^{\prime}}\left(m, v_{m}^{-\left(i, j^{\prime}\right)}, S\right) .
$$


The second axiom of permission fairness considers the payoff variations of the first activity level of a player $k$ and the maximal activity level of its superior $i$ when player $k$ and all its subordinates do not participate anymore. This axiom requires that the payoff variations of these two activity-level pairs are the same.

Axiom 7 (Inter m-fairness). For each $(m, v, S) \in \mathcal{G}^{\mathcal{T}}$ and each $(k, 1) \in M^{+}$, where $k$ is different from the root of $S$, and $i \in S^{-1}(k)$, it holds that:

$$
f_{i m_{i}}(m, v, S)-f_{i m_{i}}\left(m, v_{m}^{-(k, 1)}, S\right)=f_{k 1}(m, v, S)-f_{k 1}\left(m, v_{m}^{-(k, 1)}, S\right) .
$$

In a pal-permission structure $S_{m}^{+}$, a coalition $s \in \mathcal{M}$ is feasible in the sense of (6) if the superiors of each active player have reached their maximal activity level. From this observation, the set of feasible coalitions $\mathcal{F}\left(m, S_{m}^{+}\right)$is given by

$$
\mathcal{F}\left(m, S_{m}^{+}\right):=\left\{s \in M:\left[s_{i}>0 \text { and } k \in \hat{S}^{-1}(i)\right] \Longrightarrow\left[s_{k}=m_{k}\right]\right\}
$$

from which we deduce the following lemma.

Lemma 5. The sovereign part $\sigma(s)$ and the authorizing part $\alpha(s)$ of a coalition $s$ with respect to $S_{m}^{+}$are as follow $\S^{1}$.

$$
\begin{aligned}
& -\forall i \in N, \quad \sigma_{i}(s)= \begin{cases}0 & \text { if there exists } k \in \hat{S}^{-1}(i) \text { such that } s_{k}<m_{k}, \\
s_{i} & \text { else. }\end{cases} \\
& \text { - } \forall i \in N, \quad \alpha_{i}(s)= \begin{cases}m_{i} & \text { if there exists } k \in \hat{S}(i) \text { such that } s_{k}>0, \\
s_{i} & \text { else. }\end{cases}
\end{aligned}
$$

Proof. The proof follows directly from (15) and (7).

Example 5. In Example 3, the set of feasible coalitions under $S_{m}^{+}$consists of all coalitions such that if $s_{W}>0$, then $s_{F}=3$. For instance, coalition $\left(s_{F}, s_{W}\right)=(2,2)$ is not feasible since $s_{W}=2>0$ and $s_{F}=2<3$. In this case, $\sigma(2,2)=(2,0)$ because $\sigma_{W}(2,2)=0$ for the above mentioned reason. On the other hand, $\alpha(2,2)=(3,2)$ since $\alpha_{F}(2,2)=3$.

The next lemma identifies a subset of coalitions whose worth is unchanged in the associated $(k, l)$-game $\left(m, v_{m}^{-(k, l)}, S\right)$. From this lemma, we deduce that the Harsanyi dividends of these coalitions coincide in $(m, v, S)$ and $\left(m, v_{m}^{-(k, l)}, S\right)$.

Lemma 6. For each $(m, v, S) \in \mathcal{G}^{\mathcal{T}}$, each $(k, l) \in M^{+}$and each coalition $s \in \mathcal{M}$ such that $\alpha_{k}(s)<l$, it holds that:

$$
v_{m}^{-(k, l)}(s)=v(s) .
$$

\footnotetext{
${ }^{1}$ We continue to denote the sovereign part and the authorizing part of $s$ by $\sigma(s)$ and $\alpha(s)$ with the understanding that the underlying permission structure is $S_{m}^{+}$.
} 
Proof. By definition of $v_{m}^{-(k, l)}(s)$,

$$
v_{m}^{-(k, l)}(s)=v(\bar{s}),
$$

where

$$
\bar{s}_{h}= \begin{cases}l-1 & \text { if } h=k \text { and } s_{h} \geq l, \\ 0 & \text { if } h \in \hat{S}(k), \\ s_{h} & \text { else. }\end{cases}
$$

First, by Lemma 5, $\alpha_{k}(s)<l \leq m_{i}$ and $h \in \hat{S}(k)$ imply that $s_{h}=0$, so that $s_{h}=\bar{s}_{h}=0$. Second, by definition of the authorizing part of a coalition, $s_{k} \leq \alpha_{k}(s)$. Thus, by definition of $v_{m}^{-(k, l)}, \bar{s}_{k}=s_{k}$. Third, by definition of $v_{m}^{-(k, l)}$, we also have $\bar{s}_{h}=s_{h}$ for each other player $h \notin \hat{S}(k) \cup\{k\}$. Conclude that $\bar{s}=s$ and so $v_{m}^{-(k, l)}(s)=v(\bar{s})=v(s)$.

Lemma 7. For each $(m, v, S) \in \mathcal{G}^{\mathcal{T}}$ and each $s \in \mathcal{M}$ such that $\alpha_{k}(s)<l$, it holds that:

$$
\Delta_{v_{m}^{-(k, l)}}(s)=\Delta_{v}(s) .
$$

Proof. The proof follows from Lemma 6 and (1).

We have the material to provide the main result of this subsection.

Theorem 2. An allocation rule $f$ satisfies Efficiency, the m-inessential pair property, Intra $m$-fairness and Inter m-fairness on $\mathcal{G}^{\mathcal{T}}$ if and only if $f=f^{(+, m)}$.

Proof. First, we show that $f^{(+, m)}$ satisfies all the axioms of the statement of Theorem 7 . Consider any $(m, v, S) \in \mathcal{G}^{\mathcal{T}}$. The permission structure $S$ induces the unique m-permission structure $S_{m}^{+} \in \mathcal{A}^{+}$and thus a unique game $\left(m, v, S_{m}^{+}\right) \in \mathcal{G}^{\mathcal{A}^{+}}$.

Efficiency: by definition of $f^{(+, m)}$,

$$
f^{(+, m)}(m, v, S)=D P\left(m, \mathcal{R}_{S_{m}^{+}}(v)\right)=D P(m, v \circ \sigma) .
$$

From Klijn et al. [1999], we know that the $D P$ value is an efficient value, and from (7), we know that $\sigma(m)=m$. Thus, we get:

$$
\sum_{i \in N} \sum_{j=1}^{m_{i}} f_{i j}^{(+, m)}(m, v, S)=\sum_{i \in N} \sum_{j=1}^{m_{i}} D P_{i j}(v \circ \sigma)=v(\sigma(m))=v(m),
$$

which proves that $f^{(+, m)}$ satisfies Efficiency.

m-inessential pair property: From Klijn et al. [1999], we know that the $D P$ value satisfies the dummy level property, that is, each dummy pair obtains a null payoff in a multi-choice game. Thus, by definition of $f^{(+, m)}$, it is sufficient to prove that a $\mathrm{m}$ inessential pair in $(m, v, S)$ is a dummy pair in $\left(m, \mathcal{R}_{S_{m}^{+}}(v)\right)$. Consider any m-inessential pair $(i, j) \in I L^{m}(m, v, S)$ and any coalitions $s, s^{\prime} \in \mathcal{M}$ such that $s_{i}=j-1$ and $s^{\prime}=\left(s_{-i}, l\right)$, $j \leq l \leq m_{i}$. Because $s_{k}^{\prime}=s_{k}$ for each $k \in N \backslash \hat{S}(i)$, it follows that $\sigma_{k}\left(s^{\prime}\right)=\sigma_{k}(s)$. For each $k \in \hat{S}(i) \cup\{i\}$, we have $\sigma_{k}\left(s^{\prime}\right) \geq \sigma_{k}(s)$. In particular, the superiors of player $i$ play the same activity level in $s$ and $s^{\prime}$. Thus, if $\sigma_{i}(s)=0$, then $\sigma_{i}\left(s^{\prime}\right)=0$, and so $v\left(\sigma\left(s^{\prime}\right)\right)=v(\sigma(s))$. 
If $\sigma_{i}(s)=j-1$, then $\sigma_{i}\left(s^{\prime}\right)=l \geq j$. Because $(i, j)$ is a m-inessential pair, we still have $v\left(\sigma\left(s^{\prime}\right)\right)=v(\sigma(s))$. It follows that $(i, j)$ is a dummy pair in $\mathcal{R}_{S_{m}^{+}}(v)$, and so

$$
f_{i j}^{(+, m)}(m, v, S)=D P_{i j}\left(m, \mathcal{R}_{S_{m}^{+}}(v)\right)=0,
$$

which shows that $f^{(+, m)}$ satisfies the m-inessential pair property.

Intra m-fairness: Consider any two pairs $(i, j),\left(i, j^{\prime}\right) \in M^{+}$such that $j<m_{i}$ and $j^{\prime}>j$. By definition, it holds that:

$$
\begin{aligned}
& f_{i j}^{(+, m)}(m, v, S)-f_{i j}^{(+, m)}\left(m, v_{m}^{-\left(i, j^{\prime}\right)}, S\right) \\
& =\sum_{\substack{s \in \mathcal{M} \\
j \leq \alpha_{i}(s)}} \frac{\Delta_{v}(s)}{\sum_{h \in N} \alpha_{h}(s)}-\sum_{\substack{s \in \mathcal{M}\left(j \leq \alpha_{i}(s) \\
j\right.}} \frac{\Delta_{v_{m}^{-\left(i, j^{\prime}\right)}}(s)}{\sum_{h \in N} \alpha_{h}(s)} \\
& =\sum_{\substack{s \in \mathcal{M} \\
j \leq \alpha_{i}(s)}} \frac{\Delta_{v}(s)}{\sum_{h \in N} \alpha_{h}(s)}-\sum_{\substack{s \in \mathcal{M} \\
j \leq \alpha_{i}(s) \\
j^{\prime}>\alpha_{i}(s)}} \frac{\Delta_{\left.v_{m}^{-\left(i, j^{\prime}\right.}\right)}(s)}{\sum_{h \in N} \alpha_{h}(s)}-\sum_{\substack{s \in \mathcal{M} \\
j \leq \alpha_{i}(s) \\
j^{\prime} \leq \alpha_{i}(s)}} \frac{\Delta_{v_{m}^{-\left(i, j^{\prime}\right)}}(s)}{\sum_{h \in N} \alpha_{h}(s)}
\end{aligned}
$$

By Lemma 7, $\Delta_{v_{m}^{-\left(i, j^{\prime}\right)}}(s)=\Delta_{v}(s)$ when $\alpha_{i}(s)<j^{\prime}$. It follows that:

$$
\begin{aligned}
& f_{i j}^{(+, m)}(m, v, S)-f_{i j}^{(+, m)}\left(m, v_{m}^{-\left(i, j^{\prime}\right)}, S\right) \\
& =\sum_{\substack{s \in \mathcal{M} \\
j \leq \alpha_{i}(s)}} \frac{\Delta_{v}(s)}{\sum_{h \in N} \alpha_{h}(s)}-\sum_{\substack{s \in \mathcal{M} \\
j \leq \alpha_{i}(s)}} \frac{\Delta_{v}(s)}{\sum_{h \in N} \alpha_{h}(s)}-\sum_{\substack{s \in \mathcal{M} \\
j^{\prime}>\alpha_{i}(s)}} \frac{\Delta_{\left.v_{m}^{-\left(i, j^{\prime}\right.}\right)}(s)}{\sum_{h \in N} \alpha_{h}(s)} \\
& =\sum_{\substack{s \in \mathcal{M}(s) \\
j^{\prime} \leq \alpha_{i}(s)}} \frac{\Delta_{v}(s)}{\sum_{h \in N} \alpha_{h}(s)}-\sum_{\substack{s \in \mathcal{M}(s) \\
j^{\prime} \leq \alpha_{i}(s)}} \frac{\Delta_{v_{m}^{-\left(i, j^{\prime}\right)}}(s)}{\sum_{h \in N} \alpha_{h}(s)} \\
& =\sum_{\substack{s \in \mathcal{M}(s) \\
j^{\prime} \leq \alpha_{i}(s)}} \frac{\Delta_{v}(s)}{\sum_{h \in N} \alpha_{h}(s)}-\sum_{\substack{s \in \mathcal{M} \\
j^{\prime} \leq \alpha_{i}(s)}} \frac{\Delta_{v_{m}^{-\left(i, j^{\prime}\right)}}(s)}{\sum_{h \in N} \alpha_{h}(s)} \\
& =f_{i j^{\prime}}^{(+, m)}(m, v, S)-f_{i j^{\prime}}^{(+, m)}\left(m, v_{m}^{-\left(i, j^{\prime}\right)}, S\right),
\end{aligned}
$$

which shows that $f^{(+, m)}$ satisfies Intra m-fairness. In particular, $f_{i j^{\prime}}^{(+, m)}\left(m, v_{m}^{-\left(i, j^{\prime}\right)}, S\right)=0$ since $\left(i, j^{\prime}\right)$ is a m-inessential pair in $v_{m}^{-\left(i, j^{\prime}\right)}$. 
Inter m-fairness: Consider any $k, i \in N$ such that $i \in S^{-1}(k)$. By definition,

$$
\begin{aligned}
& f_{i m_{i}}^{(+, m)}(m, v, S)-f_{i m_{i}}^{(+, m)}\left(m, v_{m}^{-(k, 1)}, S\right) \\
& =\sum_{\substack{s \in \mathcal{M} \\
m_{i}=\alpha_{i}(s)}} \frac{\Delta_{v}(s)}{\sum_{h \in N} \alpha_{h}(s)}-\sum_{\substack{s \in \mathcal{M} \\
m_{i}=\alpha_{i}(s)}} \frac{\Delta_{v_{m}^{-(k, 1)}}(s)}{\sum_{h \in N} \alpha_{h}(s)} \\
& =\sum_{\substack{s \in \mathcal{M} \\
m_{i}=\alpha_{i}(s)}} \frac{\Delta_{v}(s)}{\sum_{h \in N} \alpha_{h}(s)}-\sum_{\substack{s \in \mathcal{M} \\
m_{i}=\alpha_{i}(s) \\
\alpha_{k}(s)=0}} \frac{\Delta_{v_{m}^{-(k, 1)}}(s)}{\sum_{h \in N} \alpha_{h}(s)}-\sum_{\substack{s \in \mathcal{M} \\
m_{i}=\alpha_{i}(s) \\
1 \leq \alpha_{k}(s)}} \frac{\Delta_{v_{m}^{-(k, 1)}}(s)}{\sum_{h \in N} \alpha_{h}(s)}
\end{aligned}
$$

By Lemma 7, $\Delta_{v_{m}^{-(k, 1)}}(s)=\Delta_{v}(s)$ when $\alpha_{k}(s)=0$. It follows that:

$$
\begin{aligned}
& f_{i m_{i}}^{(+, m)}(m, v, S)-f_{i m_{i}}^{(+, m)}\left(v_{m}^{-(k, 1)}, S\right) \\
& =\sum_{\substack{s \in \mathcal{M} \\
m_{i}=\alpha_{i}(s)}} \frac{\Delta_{v}(s)}{\sum_{h \in N} \alpha_{h}(s)}-\sum_{\substack{s \in \mathcal{M} \\
m_{i}=\alpha_{i}(s) \\
\alpha_{k}(s)=0}} \frac{\Delta_{v}(s)}{\sum_{h \in N} \alpha_{h}(s)}-\sum_{\substack{s \in \mathcal{M} \\
m_{i}=\alpha_{i}(s) \\
1 \leq \alpha_{k}(s)}} \frac{\Delta_{v_{m}^{-(k, 1)}}(s)}{\sum_{h \in N} \alpha_{h}(s)} \\
& =\sum_{\substack{s \in \mathcal{M} \\
m_{i}=\alpha_{i}(s) \\
1 \leq \alpha_{k}(s)}} \frac{\Delta_{v}(s)}{\sum_{h \in N} \alpha_{h}(s)}-\sum_{\substack{s \in \mathcal{M} \\
m_{i}=\alpha_{i}(s) \\
1 \leq \alpha_{k}(s)}} \frac{\Delta_{v_{m}^{-(k, 1)}}(s)}{\sum_{h \in N} \alpha_{h}(s)} .
\end{aligned}
$$

Recall that, for each $s \in \mathcal{M}, \alpha(s)$ is a feasible coalition. By definition of a feasible coalition, if $\alpha_{k}(s) \geq 1>0$ then $\alpha_{i}(s)=m_{i}$ because $k \in S(i)$. It follows that:

$$
\begin{aligned}
& f_{i m_{i}}^{(+, m)}(m, v, S)-f_{i m_{i}}^{(+, m)}\left(v_{m}^{-(k, 1)}, S\right) \\
& =\sum_{\substack{s \in \mathcal{M} \\
1 \leq \alpha_{k}(s)}} \frac{\Delta_{v}(s)}{\sum_{h \in N} \alpha_{h}(s)}-\sum_{\substack{s \in \mathcal{M} \\
1 \leq \alpha_{k}(s)}} \frac{\Delta_{v_{m}^{-(k, 1)}}(s)}{\sum_{h \in N} \alpha_{h}(s)} \\
& =f_{k 1}^{(+, m)}(m, v, S)-f_{k 1}^{(+, m)}\left(m, v_{m}^{-(k, 1)}, S\right),
\end{aligned}
$$

which shows that $f^{(+, m)}$ satisfies Inter m-fairness. In particular, $f_{k 1}^{(+, m)}\left(m, v_{m}^{-(k, 1)}, S\right)=0$, because $(k, 1)$ is a m-inessential pair in $v_{m}^{-(k, 1)}$.

To complete the proof, it remains to show that there is at most one allocation rule $f$ satisfying Efficiency, the m-inessential pair property, Intra m-fairness and Inter m-fairness. So, pick any allocation rule $f$ that satisfies the above four axioms and consider any $(m, v, S) \in$ $\mathcal{G}^{\mathcal{T}}$. Without loss of generality, suppose that $2 \in S(1)$ and 1 is the root of the permission tree. To show uniqueness, we proceed by (descending) induction over the cardinality of $I L^{m}(m, v, S)$.

Initialization: If $\left|I L^{m}(m, v, S)\right|=\sum_{i \in N} m_{i}$, then each activity level of each player is a m-inessential pair. By the m-inessential pair property, it holds that $f_{i j}(m, v, S)=0$ for each 
$(i, j) \in M^{+}$. If $\left|I L^{m}(m, v, S)\right|=\sum_{i \in N} m_{i}-1$, then each activity level of each player is a minessential pair, except the first activity level of the top player, that is, $(1,1) \notin I L^{m}(m, v, S)$. Indeed, if $(1,1) \in I L^{m}(m, v, S)$ then, by definition of a m-essential pair, any pair in $M^{+}$is a dummy pair, which leads to a contradiction. By Efficiency and the m-inessential pair property, it follows that $f_{11}(m, v, S)=v(m)$ and $f_{i j}(m, v, S)=0$ for each other pair $(i, j) \neq$ $(1,1)$. So, $f$ is uniquely determined.

Induction hypothesis: suppose that the statement is true for any $(m, v, S) \in \mathcal{G}^{\mathcal{T}}$ such that $\left|I L^{m}(m, v, S)\right|=I+1$ where $I+1 \leq \sum_{i \in N} m_{i}-1$.

Induction step: pick any $(m, v, S) \in \mathcal{G}^{\mathcal{T}}$ such that $\left|I L^{m}(m, v, S)\right|=I$.

For any pair $(i, j) \in I L^{m}(m, v, S)$, the m-inessential pair property implies that:

$$
f_{i j}(m, v, S)=0 .
$$

For any two pairs $(i, j),(i, j+1) \in M_{i}^{+} \backslash I L^{m}(m, v, S)$ such that $i \in N$ and $j<m_{i}$, Intra m-fairness implies the following equation:

$$
f_{i j}(m, v, S)-f_{i(j+1)}(m, v, S)=f_{i j}\left(m, v_{m}^{-(i, j+1)}, S\right)-f_{i(j+1)}\left(m, v_{m}^{-(i, j+1)}, S\right) .
$$

Next, for any two pairs $\left(i, m_{i}\right),(k, 1) \in M^{+} \backslash I L^{m}(m, v, S)$ such that $k \in S(i)$, Inter m-fairness implies the following linear equation:

$$
f_{i m_{i}}(m, v, S)-f_{i 1}\left(m, v_{m}, S\right)=f_{i m_{i}}\left(m, v_{m}^{-(k, 1)}, S\right)-f_{k 1}\left(m, v_{m}^{-(k, 1)}, S\right) .
$$

Using Intra m-fairness and Inter $\mathrm{m}$-fairness and the fact that $S$ is a permission tree structure, we can generate $\sum_{i \in N} m_{i}-\left|I L^{m}(m, v, S)\right|-1$ linear equations. By Efficiency, we have the linear equation:

$$
\sum_{(i, j) \in M^{+} \backslash I L^{m}(m, v, S)} f_{i j}(m, v, S)=v(m) .
$$

We obtain a system of $\sum_{i \in N} m_{i}-\left|I L^{m}(m, v, S)\right|$ linear equations of the following form:

$$
\left\{\begin{array}{l}
f_{11}(m, v, S)-f_{12}(m, v, S)=f_{11}\left(m, v_{m}^{(1,2)}, S\right)-f_{12}\left(m, v_{m}^{-(1,2)}, S\right) \\
f_{12}(m, v, S)-f_{13}(m, v, S)=f_{12}\left(m, v_{m}^{(1,3)}, S\right)-f_{13}\left(m, v_{m}^{-(1,3)}, S\right) \\
\ldots \\
f_{1\left(m_{1}-1\right)}(m, v, S)-f_{1 m_{1}}(m, v, S)=f_{1\left(m_{1}-1\right)}\left(m, v_{m}^{\left(1, m_{1}\right)}, S\right)-f_{1\left(m_{1}-1\right)}\left(m, v_{m}^{-\left(1,\left(m_{1}-1\right)\right)}, S\right) \\
f_{1 m_{1}}(m, v, S)-f_{21}(m, v, S)=f_{1 m_{1}}\left(m, v_{m}^{-(2,1)}, S\right)-f_{21}\left(m, v_{m}^{-(2,1)}, S\right) \\
f_{21}(m, v, S)-f_{22}(m, v, S)=f_{21}\left(m, v_{m}^{(2,2)}, S\right)-f_{22}\left(m, v_{m}^{-(2,2)}, S\right) \\
\ldots \\
\sum_{(i, j) \in M^{+} \backslash I L^{m}(m, v, S)} f_{i j}(m, v, S)=v(m) .
\end{array}\right.
$$

Recall that $(k, 1)$ and $(i, j+1)$ are m-inessential pairs in $v_{m}^{-(k, 1)}$ and $v_{m}^{-(i, j+1)}$ respectively, but are not m-inessential pairs in $v$. It follows that $\left|I L^{m}\left(m, v_{m}^{-(k, 1)}, S\right)\right| \geq I+1$ and 
$\left|I L^{m}\left(v_{m}^{-(i, j+1)}, S\right)\right| \geq I+1$. By the induction hypothesis, $f\left(m, v_{m}^{-(k, 1)}, S\right)$ and $f\left(m, v_{m}^{-(i, j+1)}, S\right)$ are uniquely determined. Therefore, all the unknowns of the linear system formed by (16), (17), and (18) are located on the left side of the equalities.

Let us show that this system of $\sum_{i \in N} m_{i}-\left|I L^{m}(m, v, S)\right|$ linear equations with $\sum_{i \in N} m_{i}-$ $\left|I L^{m}(m, v, S)\right|$ unknowns leads to a unique solution. It admits a unique solution if and only if $A x=b$ admits a unique solution $x \in \mathbb{R}^{\sum_{i \in N} m_{i}-\left|I L^{m}(m, v, S)\right|}$, where $A$ is a $\left(\sum_{i \in N} m_{i}-\right.$ $\left.\left|I L^{m}(m, v, S)\right|\right) \times\left(\sum_{i \in N} m_{i}-\left|I L^{m}(m, v, S)\right|\right)$ matrix and $b \in \mathbb{R}^{\sum_{i \in N} m_{i}-\left|I L^{m}(m, v, S)\right|}$ :

$$
A=\left(\begin{array}{cccccc}
1 & -1 & 0 & \ldots & 0 & 0 \\
0 & 1 & -1 & \ldots & 0 & 0 \\
\ldots & & & & & \\
0 & 0 & \ldots & 1 & -1 & 0 \\
0 & 0 & \ldots & 0 & 1 & -1 \\
1 & 1 & 1 & \ldots & 1 & 1
\end{array}\right) \quad b=\left(\begin{array}{c}
f_{11}\left(m, v_{m}^{(1,2)}, S\right)-f_{12}\left(m, v_{m}^{-(1,2)}, S\right) \\
f_{12}\left(m, v_{m}^{(1,3)}, S\right)-f_{13}\left(m, v_{m}^{-(1,3)}, S\right) \\
\ldots \\
f_{1 m_{1}}\left(m, v_{m}^{-(2,1)}, S\right)-f_{21}\left(m, v_{m}^{-(2,1)}, S\right) \\
\ldots \\
v(m)
\end{array}\right)
$$

And $A x=b$ admits a unique solution $x=A^{-1} b$ if and only if $A$ is invertible, i.e., $A$ is full rank.

We show that $A$ is full rank. We prove such statement by showing the linear independence of $A$ 's columns. Let us denote by $a_{i}$ the $i$-th column of $A$ such that $A=\left(a_{1}, \ldots, a_{n}\right)$. Suppose that there exists $\lambda \in \mathbb{R}^{\sum_{i \in N} m_{i}-\left|I L^{m}(m, v, S)\right|}$ such that

$$
\lambda_{1} a_{1}+\lambda_{2} a_{2}+\ldots+\lambda_{\sum_{i \in N} m_{i}-\left|I L^{m}(m, v, S)\right|} a_{\sum_{i \in N} m_{i}-\left|I L^{m}(m, v, S)\right|}=0 .
$$

First, one can infer from the $\sum_{i \in N} m_{i}-\left|I L^{m}(m, v, S)\right|-1$ first rows of $A$ that

$$
\lambda_{1}=\lambda_{2}=\ldots=\lambda_{\sum_{i \in N} m_{i}-\left|I L^{m}(m, v, S)\right|} .
$$

Then, the last row of $A$ indicates that $\lambda_{1}+\lambda_{2}+\ldots+\lambda_{\sum_{i \in N} m_{i}-\left|I L^{m}(m, v, S)\right|}=0$. Hence $\lambda_{1}=\lambda_{2}=\ldots=\lambda_{\sum_{i \in N} m_{i}-\left|I L^{m}(m, v, S)\right|}=0$, meaning the columns of $A$ are linearly independent which is equivalent to say that $A$ is a full rank matrix. Hence, the linear system of equations leads to a unique solution. This complete the proof of the induction step.

Conclude that $f^{(+, m)}$ is the unique allocation rule on $\mathcal{G}^{\mathcal{T}}$ satisfying Efficency the minessential pair property, Intra m-fairness and Inter m-fairness, as desired.

\subsection{Characterization of $f^{(+, 1)}$}

The reasoning is similar to the previous one, except that the notions of inessential players and fairness properties must be adjusted to take into account the pal-permission structure $S^{+, 1}$.

Here, a player is considered to be inessential in two cases. First, if it and each of its subordinates do not generate any worth at any level of activity. Second, if there is an activity level greater than 1 at which its productivity falls to zero. Formally, a pair $(i, j) \in M^{+}$is a 1-inessential pair in $(m, v, S) \in \mathcal{G}^{\mathcal{T}}$ in the following two cases:

- either $j=1$ and $(i, j)$ is a dummy pair in $(m, v, S)$ and for each $k \in \hat{S}(i),(k, 1)$ is also a dummy pair in $(m, v, S)$; 
- or $j>1$ and $(i, j)$ is a dummy pair in $(m, v, S)$.

The set of 1 -inessential pairs in $(m, v, S)$ is denoted by $I L^{1}(m, v, S)$. Observe that $I L^{m}(m, v, S) \subseteq I L^{1}(m, v, S)$. This leads to a new inessential pair property, weaker than the m-inessential pair property.

Axiom 8 (1-inessential pair property). For each $(m, v, S) \in \mathcal{G}^{\mathcal{T}}$, if $(i, j) \in I L^{1}(m, v, S)$, then it holds that:

$$
f_{i j}(m, v, S)=0 .
$$

Next, we provide two new axioms of intra and inter fairness, which are based on similar principles as the previous ones except that the associated game from which they are defined is different. Precisely, consider any $(m, v, S) \in \mathcal{G}^{\mathcal{T}}$. For each pair $\left(i, j^{\prime}\right) \in M^{+}$, define the associated $\left(i, j^{\prime}\right)$-game $\left(m, v_{1}^{-\left(i, j^{\prime}\right)}, S\right) \in \mathcal{G}^{\mathcal{T}}$ where in $v_{1}^{-\left(i, j^{\prime}\right)}$, player $i$ stops its activity from level $j^{\prime}$ onwards. If $j^{\prime}>1$, the other players are not affected. If $j=1$, then $i$ and its subordinates are no longer active. For each $s \in \mathcal{M}$, we have:

$$
v_{1}^{-\left(i, j^{\prime}\right)}(s)=v(\tilde{s}) \quad \text { where } \tilde{s}_{k}= \begin{cases}j^{\prime}-1 & \text { if } k=i \text { and } s_{k} \geq j^{\prime} \\ 0 & \text { if } k \in \hat{S}(i) \text { and } j^{\prime}=1 \\ s_{k} & \text { else }\end{cases}
$$

In $\left(m, v_{1}^{-(i, j)}, S\right)$ two cases occur: either $j^{\prime}=1$, and in this case any pair $(k, l)$ such that $k \in \hat{S}(i) \cup\{i\}$ becomes a 1-inessential pair; or $j^{\prime}>1$, and in this case, for each $j \geq j^{\prime}$, the pair $(i, j)$ becomes 1-inessential.

Axiom 9 (Intra 1-fairness). For each $(m, v, S) \in \mathcal{G}^{\mathcal{T}}$ and each $(i, j),\left(i, j^{\prime}\right) \in M^{+}$such that $j^{\prime} \geq j$, it holds that:

$$
f_{i j}(m, v, S)-f_{i j}\left(m, v_{1}^{-\left(i, j^{\prime}\right)}, S\right)=f_{i j^{\prime}}(m, v, S)-f_{i j^{\prime}}\left(m, v_{1}^{-\left(i, j^{\prime}\right)}, S\right) .
$$

Axiom 10 (Inter 1-fairness). For each $(m, v, S) \in \mathcal{G}^{\mathcal{T}}$, each $k \in N$, each $(k, 1) \in M^{+}$and each $i \in S^{-1}(k)$, it holds that:

$$
f_{i 1}(m, v, S)-f_{i 1}\left(m, v_{1}^{-(k, 1)}, S\right)=f_{k 1}(m, v, S)-f_{k 1}\left(m, v_{1}^{-(k, 1)}, S\right) .
$$

Contrary to Intra m-fairness, which compares the variations in payoffs of $(k, 1)$ and $\left(i, m_{i}\right)$, Inter 1-fairness compares the variations in payoffs of $(k, 1)$ and $(i, 1)$.

In a pal-permission structure $S_{1}^{+}$, a coalition $s \in \mathcal{M}$ is feasible if the superiors of each active player are active as well. From this observation the set of feasible coalitions $\mathcal{F}\left(m, S_{1}^{+}\right)$ is given by

$$
\mathcal{F}\left(m, S_{1}^{+}\right)=\left\{s \in M:\left[s_{i}>0 \text { and } k \in S^{-1}(i)\right] \Longrightarrow\left[s_{k} \geq 1\right]\right\} .
$$

Example 6. From Example 3, the coalition $\left(s_{F}, s_{W}\right)=(2,1)$ is a feasible coalition in $S_{1}^{+}$ (see Figure (b)). In this situation, $W$ cannot cooperate until $F$ makes at least its first activity 
level. When the coalition $(2,1)$ forms, it is understood that $F$ undertakes its activity level 1 before reaching level 2. In this sense, both pairs $(F, 1)$ and $(F, 2)$ are to be considered, and because $(W, 1) \in S_{1}^{+}(F, 1), W$ is allowed to make its activity level 1 . In other words, although $(F, 2)$ is not a predecessor of $(W, 1)$ in $S_{1}^{+}$, we consider that if level 2 is reached by $F$ then level 1 must have been reached as well.

From the definition of feasible coalitions, we deduce the following lemma.

Lemma 8. The sovereign part $\sigma(s)$ and the authorizing part $\alpha(s)$ associated with a coalition $s \in \mathcal{M}$ in $S_{1}^{+}$are as follows:

- $\forall i \in N, \quad \sigma_{i}(s)= \begin{cases}0 & \text { if there exists } k \in \hat{S}^{-1}(i) \text { such that } s_{k}=0, \\ s_{i} & \text { else. }\end{cases}$

- $\forall i \in N, \quad \alpha_{i}(s)= \begin{cases}1 & \text { if there exists } k \in \hat{S}(i) \text { such that } s_{k}>0 \text { and } s_{i}=0, \\ s_{i} & \text { else. }\end{cases}$

Proof. The proof of the lemma follows directly from (19) and (7).

Example 7. Continuation of Example 6. The set of feasible coalitions under $S_{1}^{+}$consists of all coalitions such that if $s_{W}>0$, then $s_{F} \geq 1$. For instance, coalition $\left(s_{F}, s_{W}\right)=(0,1)$ is not feasible since $s_{W}=1>0$ and $s_{F}=0<1$.

The next lemma identifies a subset of coalitions whose worth is unchanged in the associated $(k, l)$-game $\left(m, v_{1}^{-(k, l)}, S\right)$. From this lemma, we obtain that the Harsanyi dividends of these coalitions coincide in $(m, v, S)$ and $\left(m, v_{1}^{-(k, l)}, S\right)$.

Lemma 9. For each $(m, v, S) \in \mathcal{G}^{\mathcal{T}}$ and each coalition $s \in M$ such that $\alpha_{k}(s)<l$, it holds that:

$$
v_{1}^{-(k, l)}(s)=v(s) .
$$

Proof. By definition of $v_{1}^{-(k, l)}$,

$$
v_{1}^{-(k, l)}(s)=v(\tilde{s})
$$

where

$$
\tilde{s}_{h}= \begin{cases}l-1 & \text { if } h=k \text { and } s_{h} \geq l, \\ 0 & \text { if } h \in \hat{S}(k) \text { and } l=1, \\ s_{h} & \text { else. }\end{cases}
$$

By definition of the authorizing part of $s$ and hypothesis, $s_{k} \leq \alpha_{k}(s)<l$. Thus, $s_{k}<l$ and so $\tilde{s}_{k}=s_{k}$. For each $h \in \hat{S}(k)$, if $l=1$, then $\alpha_{k}(s)=0=s_{k}$. Thus, by Lemma $8, s_{h}=0$ and, by definition, $\tilde{s}_{h}=0$, so that $s_{h}=\tilde{s}_{h}$. For any $h \notin \hat{S}(k) \cup\{k\}$, by definition, $\tilde{s}_{k}=s_{k}$. All in all, we get $v_{1}^{-(k, l)}(s)=\tilde{v}(s)=v(s)$.

Lemma 10. For each $(m, v, S) \in \mathcal{G}^{\mathcal{T}}$ and each $s \in M$ such that $\alpha_{k}(s)<l$, it holds that:

$$
\Delta_{v_{1}^{-(k, l)}(s)}=\Delta_{v}(s) .
$$


Theorem 3. An allocation rule $f$ satisfies Efficiency, the 1-inessential pair property, Intra 1-fairness and Inter 1-fairness on $\mathcal{G}^{\mathcal{T}}$ if and only if $f=f^{(+, 1)}$.

Proof. We only show that $f^{(+, 1)}$ satisfies the 1-inessential pair property, Intra 1-fairness and Inter 1-fairness. The rest of the proof is similar to the proof of Theorem 2 . Consider any $(m, v, S) \in \mathcal{G}^{\mathcal{T}}$. The permission structure $S$ induces the unique 1-permission structure $S_{1}^{+} \in \mathcal{A}^{+}$and thus a unique game $\left(m, v, S_{1}^{+}\right) \in \mathcal{G}^{\mathcal{A}^{+}}$.

1-inessential pair property: to show that $f^{(+, 1)}$ satisfies the axiom it is sufficient to show that any 1-inessential pair in $(m, v, S)$ is a dummy pair in $\left(m, \mathcal{R}_{S_{1}^{+}}(v)\right)$. Consider any pair $(i, j) \in I L^{1}(m, v, S)$. Pick any $s \in M$ such that $s_{i}=j-1$, and consider the coalition $s^{\prime}=\left(s_{-i}, l\right)$ such that $j \leq l \leq m_{i}$. We distinguish two cases:

- if $j=1$, then $(i, 1) \in I L^{1}(m, v, S)$ means that $(i, 1)$ is a dummy level pair as well as each pair $(k, 1), k \in \hat{S}(i)$. Next, by definition of the sovereign part of a coalition, $s^{\prime} \geq s$ implies $\sigma\left(s^{\prime}\right) \geq \sigma(s)$. By Lemma 8, for each $k \notin \hat{S}(i), \sigma_{k}\left(s^{\prime}\right)=\sigma_{k}(s)$. For $k \in \hat{S}(i) \cup\{i\}, \sigma_{k}\left(s^{\prime}\right) \geq \sigma_{k}(s)$. But, because $(i, 1) \in I L^{1}(m, v, S)$, we obtain that $v\left(\sigma\left(s^{\prime}\right)\right)=v(\sigma(s))$.

- if $j>1$, then $(i, j) \in I L^{1}(m, v, S)$ means that the pair $(i, j)$ is a dummy level pair. By Lemma 8, only the sovereign part of $(i, j)$ 's subordinates can be affected by a deviation initiated by $i$, and, by definition of $S_{1}^{+}$, these subordinates are precisely the pairs $(i, j+1), \ldots,\left(i, m_{i}\right)$. Because $j>1$, the sovereign part of $(i, j)$ 's subordinates are not affected by such a deviation. Finally, because $(i, j)$ is a dummy level pair, it follows that $v\left(\sigma\left(s^{\prime}\right)\right)=v(\sigma(s))$.

Thus, $(i, j)$ is a dummy pair in $\left(m, \mathcal{R}_{S_{1}^{+}}(v)\right)$. By definition of $f^{(+, 1)}$ and the Dummy level property applied to the DP value, we get:

$$
f_{i j}^{(+, 1)}(m, v, S)=D P_{i j}\left(m, \mathcal{R}_{S_{1}^{+}}(v)\right)=0,
$$

which shows that $f^{(+, 1)}$ satisfies the 1-inessential pair property.

Intra 1-fairness: Consider any two $(i, j),\left(i, j^{\prime}\right) \in M^{+}$such that $j<m_{i}$ and $j^{\prime}>j$. By definition of $f^{(+, 1)}$, it holds that:

$$
\begin{aligned}
& f_{i j}^{(+, 1)}(m, v, S)-f_{i j}^{(+, 1)}\left(m, v_{1}^{-\left(i, j^{\prime}\right)}, S\right) \\
& =\sum_{\substack{s \in M \\
j \leq \alpha_{i}(s)}} \frac{\Delta_{v}(s)}{\sum_{h \in N} \alpha_{h}(s)}-\sum_{\substack{s \in M \\
j \leq \alpha_{i}(s)}} \frac{\Delta_{\left.v_{1}^{-\left(i, j^{\prime}\right.}\right)}(s)}{\sum_{h \in N} \alpha_{h}(s)} \\
& =\sum_{\substack{s \in M \\
j \leq \alpha_{i}(s)}} \frac{\Delta_{v}(s)}{\sum_{h \in N} \alpha_{h}(s)}-\sum_{\substack{s \in M \\
j \leq \alpha_{i}(s) \\
j^{\prime}>\alpha_{i}(s)}} \frac{\Delta_{\left.v_{1}^{-\left(i, j^{\prime}\right.}\right)}(s)}{\sum_{h \in N} \alpha_{h}(s)}-\sum_{\substack{s \in M \\
j \leq \alpha_{i}(s) \\
j^{\prime} \leq \alpha_{i}(s)}} \frac{\Delta_{\left.v_{1}^{-\left(i, j^{\prime}\right.}\right)}(s)}{\sum_{h \in N} \alpha_{h}(s)} .
\end{aligned}
$$


By Lemma 10, $\Delta_{v_{1}^{-\left(i, j^{\prime}\right)}}=\Delta_{v}(s)$ when $\alpha_{i}(s)<j^{\prime}$. It follows that:

$$
\begin{aligned}
& f_{i j}^{(+, 1)}(m, v, S)-f_{i j}^{(+, 1)}\left(m, v_{1}^{-\left(i, j^{\prime}\right)}, S\right) \\
& =\sum_{\substack{s \in M \\
j \leq \alpha_{i}(s)}} \frac{\Delta_{v}(s)}{\sum_{h \in N} \alpha_{h}(s)}-\sum_{\substack{s \in M \\
j \leq \alpha_{i}(s) \\
j^{\prime}>\alpha_{i}(s)}} \frac{\Delta_{v}(s)}{\sum_{h \in N} \alpha_{h}(s)}-\sum_{\substack{s \in M \\
j \leq \alpha_{i}(s) \\
j^{\prime} \leq \alpha_{i}(s)}} \frac{\Delta_{\left.v_{1}^{-\left(i, j^{\prime}\right.}\right)}(s)}{\sum_{h \in N} \alpha_{h}(s)} \\
& =\sum_{\substack{s \in M \\
j \leq \alpha_{i}(s) \\
j^{\prime} \leq \alpha_{i}(s)}} \frac{\Delta_{v}(s)}{\sum_{h \in N} \alpha_{h}(s)}-\sum_{\substack{s \in M \\
j \leq \alpha_{i}(s) \\
j^{\prime} \leq \alpha_{i}(s)}} \frac{\Delta_{v_{1}^{-\left(i, j^{\prime}\right)}}(s)}{\sum_{h \in N} \alpha_{h}(s)} \\
& =\sum_{\substack{s \in M \\
j^{\prime} \leq \alpha_{i}(s)}} \frac{\Delta_{v}(s)}{\sum_{h \in N} \alpha_{h}(s)}-\sum_{\substack{s \in M \\
j^{\prime} \leq \alpha_{i}(s)}} \frac{\Delta_{\left.v_{1}^{-\left(i, j^{\prime}\right.}\right)}(s)}{\sum_{h \in N} \alpha_{h}(s)} \\
& =f_{i j^{\prime}}^{(+, 1)}(m, v, S)-f_{i j^{\prime}}^{(+, 1)}\left(m, v_{1}^{-\left(i, j^{\prime}\right)}, S\right),
\end{aligned}
$$

which shows that $f^{(+, 1)}$ satisfies Intra 1-fairness. Note that $f_{i j^{\prime}}^{(+, 1)}\left(m, v_{1}^{-\left(i, j^{\prime}\right)}, S\right)=0$ because $\left(i, j^{\prime}\right)$ is a 1-inessential pair in $\left(m, v_{1}^{-\left(i, j^{\prime}\right)}, S\right)$.

Inter 1-fairness: Consider any $k, i \in N$ such that $i \in S^{-1}(k)$. It holds that:

$$
\begin{aligned}
& f_{i 1}^{(+, 1)}(m, v, S)-f_{i 1}^{(+, 1)}\left(m, v_{1}^{-(k, 1)}, S\right) \\
& =\sum_{\substack{s \in M \\
1 \leq \alpha_{i}(s)}} \frac{\Delta_{v}(s)}{\sum_{h \in N} \alpha_{h}(s)}-\sum_{\substack{s \in M \\
1 \leq \alpha_{i}(s)}} \frac{\Delta_{v_{1}^{-(k, 1)}}(s)}{\sum_{h \in N} \alpha_{h}(s)} \\
& =\sum_{\substack{s \in M \\
1 \leq \alpha_{i}(s)}} \frac{\Delta_{v}(s)}{\sum_{h \in N} \alpha_{h}(s)}-\sum_{\substack{s \in M \\
1 \leq \alpha_{i}(s) \\
1>\alpha_{k}(s)}} \frac{\Delta_{v_{1}^{-(k, 1)}}(s)}{\sum_{h \in N} \alpha_{h}(s)}-\sum_{\substack{s \in M \\
1 \leq \alpha_{i}(s) \\
1 \leq \alpha_{k}(s)}} \frac{\Delta_{v_{1}^{-(k, 1)}}(s)}{\sum_{h \in N} \alpha_{h}(s)} .
\end{aligned}
$$


By Lemma 10, note that $\Delta_{v_{1}^{-(k, 1)}(s)}=\Delta_{v}(s)$ when $\alpha_{k}(s)<1$. It follows that:

$$
\begin{aligned}
& f_{i 1}^{(+, 1)}(m, v, S)-f_{i 1}^{(+, 1)}\left(m, v_{1}^{-(k, 1)}, S\right) \\
& =\sum_{\substack{s \in M \\
1 \leq \alpha_{i}(s)}} \frac{\Delta_{v}(s)}{\sum_{h \in N} \alpha_{h}(s)}-\sum_{\substack{s \in M \\
1 \leq \alpha_{i}(s) \\
1>\alpha_{k}(s)}} \frac{\Delta_{v}(s)}{\sum_{h \in N} \alpha_{h}(s)}-\sum_{\substack{s \in M \\
1 \leq \alpha_{i}(s) \\
1 \leq \alpha_{k}(s)}} \frac{\Delta_{v_{1}^{-(k, 1)}}(s)}{\sum_{h \in N} \alpha_{h}(s)} \\
& =\sum_{\substack{s \in M \\
1 \leq \alpha_{i}(s)}} \frac{\Delta_{v}(s)}{\sum_{h \in N} \alpha_{h}(s)}-\sum_{\substack{s \in M \\
1 \leq \alpha_{i}(s)}} \frac{\Delta_{v_{1}^{-(k, 1)}}(s)}{\sum_{h \in N} \alpha_{h}(s)} \\
& =\sum_{\substack{s \in M \\
1 \leq \alpha_{k}(s)}} \frac{\Delta_{v}(s)}{\sum_{h \in N} \alpha_{h}(s)}-\sum_{\substack{s \in M \\
1 \leq \alpha_{k}(s)}} \frac{\Delta_{v_{1}^{-(k, 1)}}(s)}{\sum_{h \in N} \alpha_{h}(s)} \\
& =f_{k 1}^{(+, 1)}(m, v, S)-f_{k 1}^{(+, 1)}\left(m, v_{1}^{-(k, 1)}, S\right)
\end{aligned}
$$

where the third equality follows from the fact that $\alpha_{k}(s) \geq 1$ implies $\alpha_{i}(s) \geq 1$ for $i \in \hat{S}^{-1}(k)$. This shows that $f^{(+, 1)}$ satisfies Inter 1-fairness. Observe that $f_{k 1}^{(+, 1)}\left(m, v_{1}^{-(k, 1)}, S\right)=0$, because $(k, 1)$ is a 1 -inessential pair in $\left(m, v_{1}^{-(k, 1)}, S\right)$.

\subsection{Characterization of $f^{(+, F)}$}

The characterization of $f^{(+, F)}$ slightly differs from the previous ones. There are two main changes.

- First, the axiomatic characterization cannot be stated on the full call of games $\mathcal{G}^{\mathcal{T}}$. Due to the definition of the F-permission structures (see section 3.4) we need to restrict the study to situations where, for each multi-choice game $(m, v)$ and each permission tree $S$, the following holds:

$$
\forall i, j \in N, \quad j \in S(i) \Longrightarrow m_{j} \leq m_{i}
$$

The class of games satisfying this assumption is denoted by $\overline{\mathcal{G}}^{\mathcal{T}}$. This class of games contains the games in which each player has the same set of activity levels. The reason for this change is that the F-permission structure conveys the idea that each player should be able to supervise its subordinates. Thus, we expect each player to be able to achieve at least the same activity levels as its subordinates.

- Second, we will no long apply the principle of permission fairness into two directions. Instead, a single axiom, generalizing the axiom of permission fairness, is proposed. As in the previous sections, the notion of inessential players is adjusted in the same way as in the previous sections. 
Here, a player is considered to be inessential if there is an activity level at which its productivity and the productivity of its subordinates fall to zero. Formally, a pair $(i, j) \in M^{+}$ is a $\mathbf{F}$-inessential pair in $(m, v, S) \in \overline{\mathcal{G}}^{\mathcal{T}}$ if it is a dummy pair in $(m, v, S)$ and if, for each $k \in \hat{S}(i),(k, j)$ is also a dummy pair in $(m, v, S)$. The set of F-inessential pairs in $(m, v, S)$ is denoted by $I L^{F}(m, v, S)$. Observe that $I L^{m}(m, v, S) \subseteq I L^{F}(m, v, S)$. This leads to a new inessential pair property, weaker than the m-inessential pair property.

Axiom 11 (F-inessential pair property). For each $(m, v, S) \in \overline{\mathcal{G}}^{\mathcal{T}}$, if $(i, j) \in I L^{F}(m, v, S)$, then it holds that:

$$
f_{i j}(m, v, S)=0 \text {. }
$$

Next, we provide one last axiom of permission fairness. Instead of dealing with permission fairness within and between players, the axiom compares the variations in payoffs of each pair with the variations in payoffs of the root of $M^{+}$. Without loss generality, we suppose that $(1,1)$ is the root of $M^{+}$. Before introducing the axiom, we need a definition. Consider any $(m, v, S) \in \overline{\mathcal{G}}^{\mathcal{T}}$. For each pair $\left(i, j^{\prime}\right) \in M^{+}$, define the associated $\left(i, j^{\prime}\right)$-game $\left(m, v_{F}^{-\left(i, j^{\prime}\right)}, S\right) \in \overline{\mathcal{G}}^{\mathcal{T}}$ where in $v_{F}^{-\left(i, j^{\prime}\right)}$, player $i$ and its subordinates stop any activity from level $j^{\prime}$ onwards. For each $s \in \mathcal{M}$, the worth $v_{F}^{-\left(i, j^{\prime}\right)}$ is defined as:

$$
v_{F}^{-\left(i, j^{\prime}\right)}(s)=v(\hat{s}), \quad \text { where } \hat{s}_{k}= \begin{cases}j^{\prime}-1 & \text { if } s_{k} \geq j^{\prime} \text { for } k \in \hat{S}(i) \cup\{i\} \\ s_{k} & \text { else. }\end{cases}
$$

By construction, for each $k \in \hat{S}(i) \cup\{i\}$ and each $l \in M_{k}^{+}$such that $l \geq j^{\prime},(k, l) \in$ $I L^{F}\left(m, v_{F}^{-\left(i, j^{\prime}\right)}, S\right)$. The next axiom of permission fairness indicates that the changes in payoffs of the pair $(1,1)$ is equal to the changes in payoffs of the pair $\left(i, j^{\prime}\right)$.

Axiom 12 (F-fairness). Consider any $(m, v, S) \in \mathcal{G}^{\mathcal{T}}$, for each $\left(i, j^{\prime}\right) \in M^{+} \backslash(1,1)$, it holds that:

$$
f_{(1,1)}(m, v, S)-f_{(1,1)}\left(m, v_{F}^{-\left(i, j^{\prime}\right), S}, S\right)=f_{\left(i, j^{\prime}\right)}(m, v, S)-f_{\left(i, j^{\prime}\right)}\left(m, v_{F}^{-\left(i, j^{\prime}\right)}, S\right)
$$

In a pal-permission structure $S_{F}^{+}$, a coalition $s \in \mathcal{M}$ is feasible in the sense of (6) if each player is active at a higher level than its subordinates. From this observation, the set of feasible coalitions according to $S_{F}^{+}$is denoted $\mathcal{F}\left(m, S_{F}^{+}\right)$and given by

$$
\mathcal{F}\left(m, S_{F}^{+}\right)=\left\{s \in M:\left[s_{i}>0 \text { and } k \in \hat{S}^{-1}(i)\right] \Longrightarrow\left[s_{k} \geq s_{i}\right]\right\}
$$

from which we deduce the following lemma.

Lemma 11. The sovereign part $\sigma(s)$ and the authorizing part $\alpha(s)$ of a coalition $s$ in $S_{F}^{+}$ are as follows:

- $\forall i \in N, \quad \sigma_{i}(s)=\min _{k \in \hat{S}^{-1}(i) \cup\{i\}} s_{k} ;$ 
- $\forall i \in N, \quad \alpha_{i}(s)=\max _{k \in \hat{S}(i) \cup\{i\}} s_{k}$.

Proof. The proof of the lemma follows directly from (20) and (7).

Example 8. Continuation of Example 6. The set of feasible coalitions under $S_{F}^{+}$consists of all coalitions such that if $s_{F} \geq s_{W}$. For instance, coalition $\left(s_{F}, s_{W}\right)=(0,1)$ is not feasible since $s_{W}=1>0$ and $s_{F}=0<1$.

As in the previous subsections, we introduce a lemma which states that the Harsanyi dividend of a certain subset of coalitions coincide in $(m, v, S)$ and $\left(m, v^{-(k, l)}, S\right)$.

Lemma 12. For each $(m, v, S) \in \overline{\mathcal{G}}^{\mathcal{T}}, s \in \mathcal{M}, k \in N$ and $l \in M_{k}^{+}$such that $\alpha_{k}(s)<l$, it holds that:

$$
\Delta_{v_{F}^{-(k, l)}}(s)=\Delta_{v}(s)
$$

We have the material to state the main result of this subsection.

Theorem 4. An allocation rule $f$ satisfies Efficiency, the F-inessential pair property and F-fairness on $\overline{\mathcal{G}}^{\mathcal{T}}$ if and only if $f=f^{(+, F)}$.

Proof. We show that $f^{(+, F)}$ satisfies the F-inessential pair property and the F-fairness. Efficiency follows in the same way as in the previous theorems. Consider any $(m, v, S) \in \overline{\mathcal{G}}^{\mathcal{T}}$. The permission structure $S$ induces the unique F-permission structure $S_{F}^{+} \in \mathcal{A}^{+}$and thus a unique game $\left(m, v, S_{F}^{+}\right) \in \mathcal{G}^{\mathcal{A}^{+}}$.

F-inessential pair property: to show that $f^{(+, F)}$ satisfies F-inessential pair property, it is sufficient to show that a F-inessential pair in $(m, v, S)$ is a dummy pair in $\left(m, \mathcal{R}_{S_{F}^{+}}(v)\right)$. Consider any pair $(i, j) \in I L^{F}(m, v, S)$. Pick any $s \in M$ such that $s_{i}=j-1$, and consider any coalition $s^{\prime}=\left(s_{-i}, l\right)$ such that $j \leq l \leq m_{i}$. By definition of the sovereign part of a coalition, $s^{\prime} \geq s$ implies $\sigma\left(s^{\prime}\right) \geq \sigma(s)$. By Lemma 11. for each $k \in \hat{S}^{-1}(i), \sigma_{k}\left(s^{\prime}\right)=\sigma_{k}(s)$, and if, for $k \in \hat{S}(i) \cup\{i\}, \sigma_{k}\left(s^{\prime}\right)>\sigma_{k}(s)$ then $\sigma_{k}\left(s^{\prime}\right)=l$. But, because $(i, j) \in I L^{F}(m, v, S)$, we obtain $v\left(\sigma\left(s^{\prime}\right)\right)=v(\sigma(s))$.

Thus, $(i, j)$ is a dummy pair in $\left(m, \mathcal{R}_{S_{F}^{+}}(v)\right)$. By definition of $f^{(+, F)}$ and the Dummy pair property apply to the $D P$ value, we get:

$$
f_{i j}^{(+, F)}(m, v, S)=D P_{i j}\left(m, \mathcal{R}_{S_{F}^{+}}(v)\right)=0,
$$

which shows that $f^{(+, F)}$ satisfies the F-inessential pair property.

F-fairness: Consider any $(k, l) \in M^{+} \backslash\{(1,1)\}$. By definition of $f^{(+, F)}$, it holds that:

$$
\begin{aligned}
& f_{11}^{(+, F)}(m, v, S)-f_{11}^{(+, F)}\left(m, v_{F}^{-(k, l)}, S\right) \\
& =\sum_{\substack{s \in M \\
1 \leq \alpha_{1}(s)}} \frac{\Delta_{v}(s)}{\sum_{h \in N} \alpha_{h}(s)}-\sum_{\substack{s \in M \\
1 \leq \alpha_{1}(s)}} \frac{\Delta_{v_{F}^{-(k, l)}}(s)}{\sum_{h \in N} \alpha_{h}(s)} \\
& =\sum_{\substack{s \in M \\
1 \leq \alpha_{1}(s)}} \frac{\Delta_{v}(s)}{\sum_{h \in N} \alpha_{h}(s)}-\sum_{\substack{s \in M \\
1 \leq \alpha_{1}(s) \\
\alpha_{k}(s)<l}} \frac{\Delta_{v_{F}^{-(k, l)}}(s)}{\sum_{h \in N} \alpha_{h}(s)}-\sum_{\substack{s \in M \\
1 \leq \alpha_{1}(s) \\
l \leq \alpha_{k}(s)}} \frac{\Delta_{v_{F}^{-(k, l)}}(s)}{\sum_{h \in N} \alpha_{h}(s)}
\end{aligned}
$$


By Lemma 12, $\Delta_{v_{F}^{-(k, l)}}(s)=\Delta_{v}(s)$ when $\alpha_{k}(s)<l$. It follows that:

$$
\begin{aligned}
& f_{11}^{(+, F)}(m, v, S)-f_{11}^{(+, F)}\left(m, v_{F}^{-(k, l)}, S\right) \\
& =\sum_{\substack{s \in M \\
1 \leq \alpha_{1}(s)}} \frac{\Delta_{v}(s)}{\sum_{h \in N} \alpha_{h}(s)}-\sum_{\substack{s \in M \\
1 \leq \alpha_{1}(s) \\
l>\alpha_{k}(s)}} \frac{\Delta_{v}(s)}{\sum_{h \in N} \alpha_{h}(s)}-\sum_{\substack{s \in M \\
1 \leq \alpha_{1}(s) \\
l \leq \alpha_{k}(s)}} \frac{\Delta_{v^{-(k, l)}}(s)}{\sum_{h \in N} \alpha_{h}(s)} \\
& =\sum_{\substack{1 \leq \alpha_{1}(s) \\
l \leq \alpha_{k}(s)}} \frac{\Delta_{v}(s)}{\sum_{h \in N} \alpha_{h}(s)}-\sum_{\substack{s \in M \\
1 \leq \alpha_{1}(s) \\
l \leq \alpha_{k}(s)}} \frac{\Delta_{v_{F}^{-}(k, l)}(s)}{\sum_{h \in N} \alpha_{h}(s)} \\
& =\sum_{\substack{s \in M \\
l \leq \alpha_{k}(s)}} \frac{\Delta_{v}(s)}{\sum_{h \in N} \alpha_{h}(s)}-\sum_{\substack{s \in M \\
l \leq \alpha_{k}(s)}} \frac{\Delta_{v_{F}^{-(k, l)}}(s)}{\sum_{h \in N} \alpha_{h}(s)} \\
& =f_{k l}^{(+, F)}(m, v, S)-f_{k l}^{(+, F)}\left(m, v_{F}^{-(k, l)}, S\right),
\end{aligned}
$$

where the third equality follows from Lemma 11 . Conclude that $f_{k l}^{(+, F)}$ satisfies F-fairness. Note that $f_{k l}^{(+, F)}\left(m, v_{F}^{-(k, l)}, S\right)=0$ because $(k, l)$ is a F-inessential pair in $\left(m, v_{F}^{-(k, l)}, S\right)$.

The uniqueness part of the proof is similar to the proof of Theorem 2 . The only difference lies in the fact that the linear system of equations arises from F-fairness and Efficiency, instead of two axioms of permission fairness and Efficiency as in the previous proofs. Indeed, remark the F-fairness generates $\sum_{i \in N} m_{i}-1$ linear equations and Efficiency generates one linear equation. We obtain a linear system of $\sum_{i \in N} m_{i}$ equations with $\sum_{i \in N} m_{i}$ unknows. Proceeding as in the proof of Theorem 2, it is easy to show that this system is linearly independent.

\section{Conclusion}

In this paper, we have considered multi-choice games with a permission tree structure. We have proposed two approaches which combine the relationships between the players with the relationships between the activity levels of each player. This led us to the definition of three specific pal-permission structures from which we have constructed three new allocation rules. We have provided comparable axiomatic characterizations of these allocations rules.

There are at least two directions of research that could be considered in the future. The first one is to consider that the players are involved in several hierarchical structures that must first be aggregated into one and then combined with the structure on the activity levels of the players. The second one is to consider that the activity levels of the players are not necessarily totally ordered but only partially ordered. Indeed, in some situations the efforts have a qualitative dimension and some types of effort are not comparable with other types of effort. 
Acknowledgments We thank Sylvain Béal, Stéphane Gonzalez, Kevin Techer and Philippe Solal for useful comments. The author wants to thank GRDF (Gaz Réseau Distribution France) for their financial support through the ANRT (Association Nationale Recherche

Technologie) doctoral program CIFRE (Conventions Industrielles de Formation par la REcherche).

\section{References}

M. J. Albizuri. The multichoice coalition value. Annals of Operations Research, 172(1):363, 2009.

R. J. Aumann and J. H. Dreze. Cooperative games with coalition structures. International Journal of Game Theory, 3(4):217-237, 1974.

S. Béal, A. Lardon, E. Rémila, and P. Solal. The average tree solution for multi-choice forest games. Annals of Operations Research, 196(1):27-51, 2012.

R. Branzei, S. Tijs, and J. Zarzuelo. Convex multi-choice games: Characterizations and monotonic allocation schemes. European Journal of Operational Research, 198(2):571575,2009 .

R. Branzei, N. Llorca, J. Sánchez Soriano, and S. Tijs. A constraint egalitarian solution for convex multi-choice games. TOP, 22(3):860-874, 2014.

E. Calvo and J. C. Santos. A value for multichoice games. Mathematical Social Sciences, 40 (3):341-354, 2000.

J. Derks and H. Peters. A Shapley value for games with restricted coalitions. International Journal of Game Theory, 21(4):351-360, 1993.

R. P. Gilles, G. Owen, and R. van den Brink. Games with permission structures: the conjunctive approach. International Journal of Game Theory, 20(3):277-293, 1992.

M. Grabisch and F. Lange. Games on lattices, multichoice games and the shapley value: a new approach. Mathematical Methods of Operations Research, 65(1):153-167, 2007.

P. J. J. Herings, G. van der Laan, and D. Talman. The average tree solution for cycle-free graph games. Games and Economic Behavior, 62(1):77-92, 2008.

C.-R. Hsiao and T. Raghavan. Monotonicity and dummy free property for multi-choice cooperative games. International Journal of Game Theory, 21(3):301-312, 1992.

F. Klijn, M. Slikker, and J. Zarzuelo. Characterizations of a multi-choice value. International Journal of Game Theory, 28(4):521-532, 1999.

R. B. Myerson. Graphs and cooperation in games. Mathematics of operations research, 2 (3):225-229, 1977. 
G. Owen. Values of games with a priori unions. In Mathematical economics and game theory, pages 76-88. Springer, 1977.

H. Peters and H. Zank. The egalitarian solution for multichoice games. Annals of Operations Research, 137(1):399-409, 2005.

L. S. Shapley. A value for n-person games. Contributions to the Theory of Games 2 (Kuhn, H. and Tucker A.W. eds), 2(28):307-317, 1953.

R. van den Brink. Games with a permission structure - a survey on generalizations and applications. TOP, 25(1):1-33, 2017.

R. van den Brink and R. P. Gilles. Axiomatizations of the conjunctive permission value for games with permission structures. Games and Economic Behavior, 12(1):113-126, 1996.

R. van den Brink, P. J.-J. Herings, G. van der Laan, and A. Talman. The average tree permission value for games with a permission tree. Economic Theory, 58(1):99-123, 2015.

R. van den Brink, C. Dietz, G. van der Laan, and G. Xu. Comparable characterizations of four solutions for permission tree games. Economic Theory, 63(4):903-923, 2017.

R. van den Brink, S. He, and J.-P. Huang. Polluted river problems and games with a permission structure. Games and Economic Behavior, 108:182-205, 2018.

C. G. A. van den Nouweland et al. Games and graphs in economic situations. PhD thesis, Tilburg University, 1993. 